Daniel Aubram, Frank Rackwitz, Stavros A. Savidis

\title{
Contribution to the Non-Lagrangian Formulation of Geotechnical and Geomechanical Processes
}

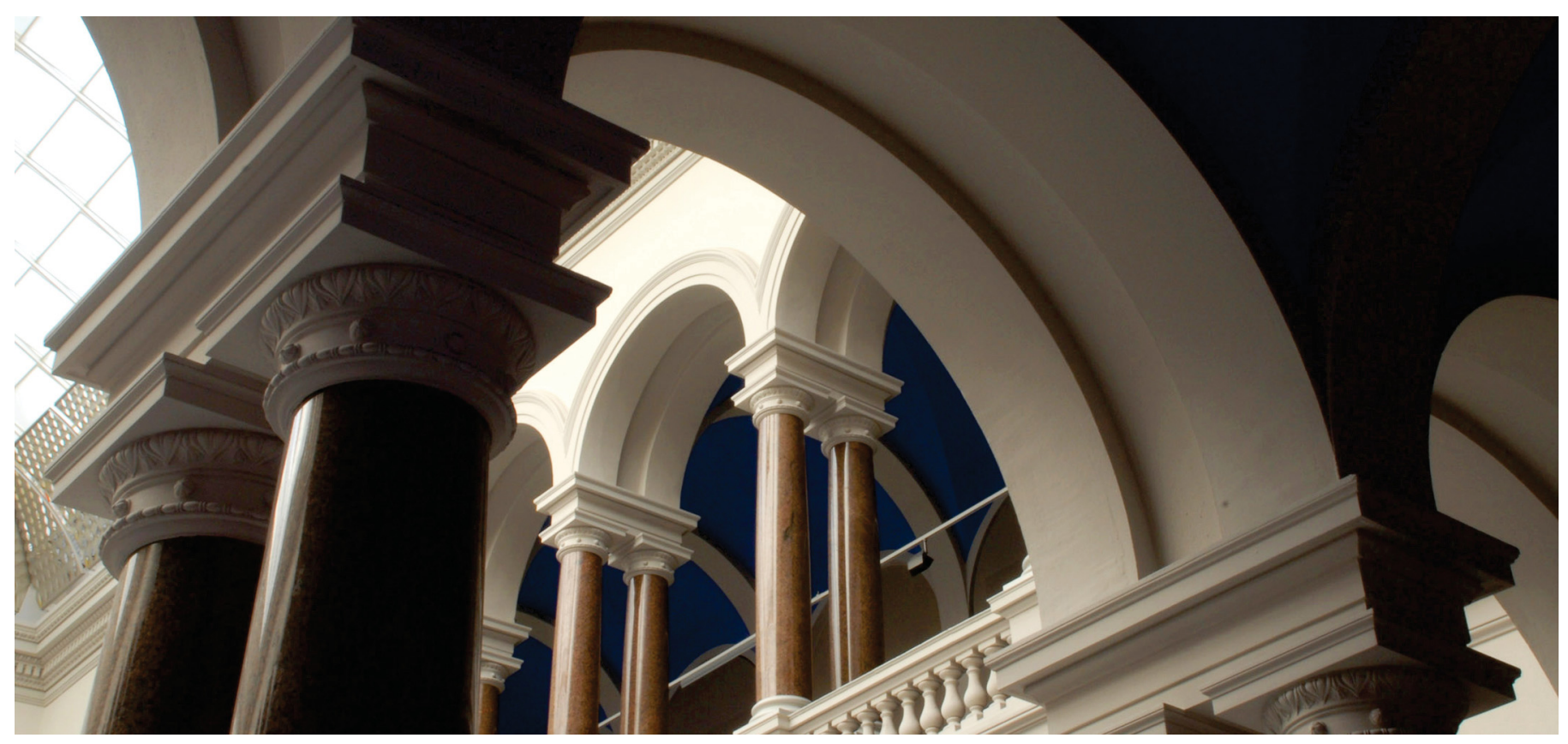

Aubram, D., Rackwitz, F., \& Savidis, S. A. (2017). Contribution to the Non-Lagrangian Formulation of Geotechnical and Geomechanical Processes. In: Triantafyllidis T. (eds) Holistic Simulation of Geotechnical Installation Processes. Lecture Notes in Applied and Computational Mechanics, vol 82. Springer, Cham. https://doi.org/10.1007/978-3-319-52590-7_3 


\title{
Contribution to the Non-Lagrangian Formulation of Geotechnical and Geomechanical Processes
}

\author{
Daniel Aubram, Frank Rackwitz, and Stavros A. Savidis \\ Chair of Soil Mechanics and Geotechnical Engineering, Technische Universität Berlin \\ (TU Berlin), Secr. TIB1-B7, Gustav-Meyer-Allee 25, D-13355 Berlin, Germany
}

\begin{abstract}
Numerical simulations of geomechanical and geotechnical processes, such as vibro-injection pile installation, require suitable algorithms and sufficiently realistic models. These models have to account for large deformations, the evolution of material interfaces including free surfaces and contact interfaces, for granular material behavior in different flow regimes as well as for the interaction of the different materials and phases. Although the traditional Lagrangian formulation is wellsuited to handling complex material behavior and maintaining material interfaces, it generally cannot represent large deformation, shear and vorticity. This is because in Lagrangian numerical methods the storage points (nodes resp. material points) move with the local material velocity, which may cause mesh tangling resp. clustering of points. The present contribution addresses the development of models for geotechnical and geomechanical processes by utilizing Eulerian and Arbitrary LagrangianEulerian (ALE) formulations. Such non-Lagrangian viewpoints introduce additional difficulties which are discussed in detail. In particular, we investigate how to track interfaces and to model interaction of different materials with respect to an arbitrarily moving control volume, and how to validate non-Lagrangian numerical models by small-scale experimental tests.
\end{abstract}

Keywords: large deformations; mixture; granular material; sand; volume averaging; closure model; interface reconstruction; Eulerian; multimaterial ALE

\section{Introduction and Literature Review}

\subsection{Geotechnical and Geomechanical Processes}

During the last decade there has been an increasing interest in gaining broad understanding of the mechanisms associated with geotechnical installation processes, and how they influence the strength and stiffness characteristics of the soil [79,155]. The main objective of the DFG Research Unit FOR 1136 GeoTech $[156,157]$, with which the authors collaborate in the context of Subproject 5 , is the provision of suitable methods for numerical simulation of such processes in order to predict the deformations of supporting systems and nearby structures.

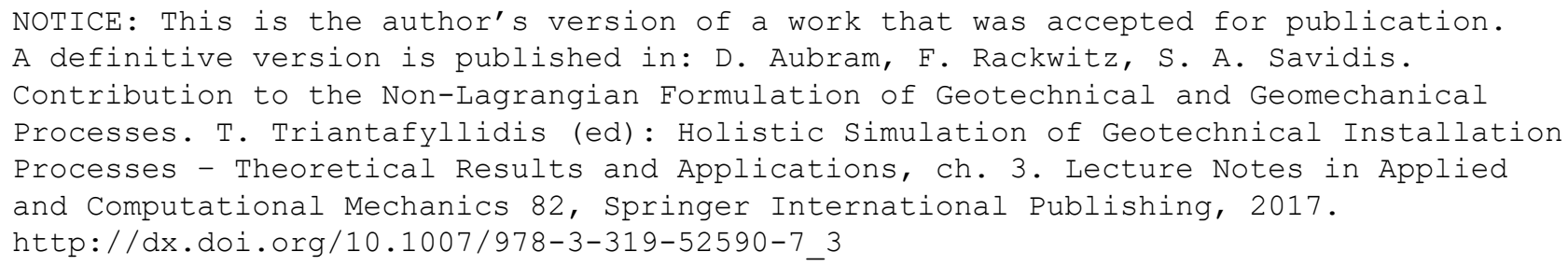


Geotechnical installation processes, like pile driving, vibro replacement, or pressure grouting, generally involve large deformations and material flow, the evolution of material interfaces including free surfaces and contact interfaces, as well as the dynamical interaction of multiple, physically distinct materials on a hierarchy of spatial scales $[14,21,118,141]$; see Fig. 1. In particular, the complexity in the behavior of the soil is attributable to its granular nature and internal structure, and to the presence of multiple phases (solid, liquid and gas). The grain-fluid mixture is generally subject to different flow regimes and undergoes changes in phase composition and internal structure depending on the dynamics of the geotechnical process $[17,22]$

The mechanisms and phenomena associated with geotechnical installation processes, except perhaps for the significance of soil-structure-interaction, are similar to those of geomechanical or geomorphological flows, for example, avalanches and debris flows [87,90,92,130,133], submarine landslides [103,114], and soil liquefaction $[97,145]$. Although the objectives of geomorphologists and geotechnical engineers in studying these phenomena may be somewhat different, both need reliable continuum mechanical models and validated numerical methods for prediction. Both also agree that multi-phase rather than single-phase or rheological approaches should be applied to capture the complexity of evolving geomaterial behavior $[87,91,176]$.

\subsection{Lagrangian Formulation}

Geotechnical engineers have been traditionally concerned with accurate determination of soil failure conditions and small deformations that may affect structures. For such situations the Lagrangian formulation of the governing equations (balance equations, constitutive models, etc.) and their discrete counterparts is well-suited because it naturally handles complex material behavior and maintains material interfaces $[57,99,144,176]$. Lagrangian formulations have also been employed to study large deformation problems in geotechnical engineering $[41,49,50,84,109]$ as well as geomechanical or granular flows $[49,90,139]$; see also [148].

The discretizations in Lagrangian methods are either mesh-based, like in the finite element method (FEM) [27,174], or point-based, like in the material point method (MPM) [25, 150] or smoothed particle hydrodynamics (SPH) [72, 105]. The major drawback of Lagrangian approaches is that they cannot represent large deformation, shear and vorticity without serious losses in accuracy and/or efficiency. This is because the storage points (mesh nodes resp. material points) move with the local material velocity, which may cause mesh distortion resp. clustering of points $[34,110]$; we remark that some point-based methods rely on a spatially fixed background mesh, but the solution variables are attributed to Lagrangian point masses. Severe local deformations may change the topology of the material, e.g. by creating new free surfaces, and thus can hardly be addressed by Lagrangian meshes without rezoning (remeshing). In such extreme 

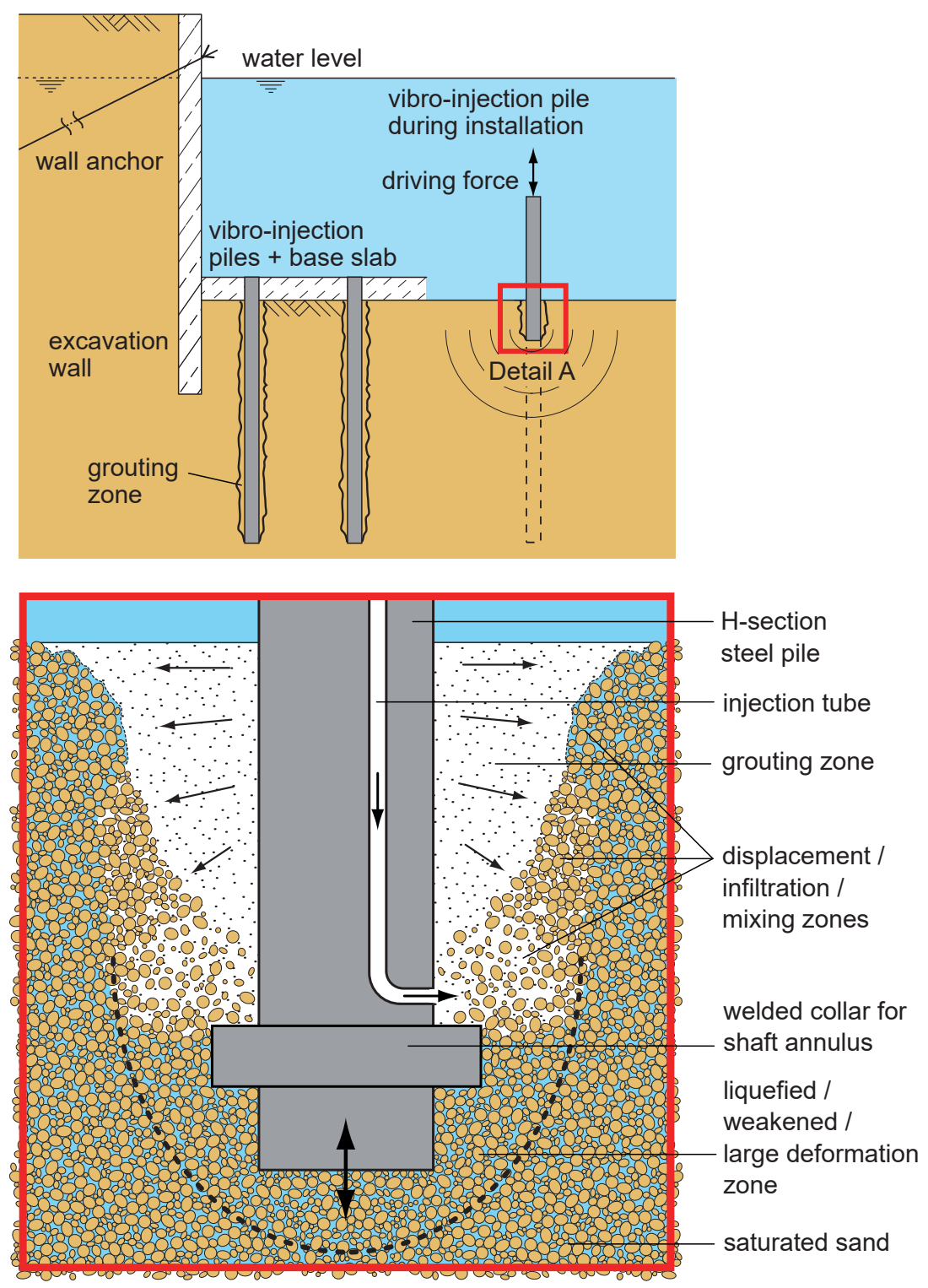

\section{Detail A: phenomenology at pile base}

Fig. 1. Schematic of a complex geotechnical process: installation of vibro-injection piles to tie back the base slab of a deep excavation. 
situations, which are likely to occur in geotechnical and geomechanical processes, calculations slow down or even terminate.

\subsection{Non-Lagrangian Formulations}

Definitions and Basic Relations In our research work we pursue a nonLagrangian approach to overcome the limitations of a Lagrangian calculation. A non-Lagrangian formulation is one where the domain of reference or a control volume moves at a velocity different from the material velocity. The reference domain is an independent continuum made up of reference points, and which is approximated by the computational mesh in numerical simulations. By definition, the mesh topology (connectivity) does not change, which distinguishes non-Lagrangian approaches from those Lagrangian techniques employing manual or automatic rezoning of the mesh.

In a non-Lagrangian approach the reference domain can be fixed in space as in the Eulerian formulation, or may move arbitrarily as in the arbitrary LagrangianEulerian (ALE) formulation [13,14,34]. Fig. 2 illustrates the different concepts. The ALE idea has been invented in the 1960-70's [82, 160, 161]. Accordingly, the spatial description of any scalar, vector or tensor field, $q$, is related to its referential or ALE description $\hat{q}$ by the composition $\hat{q}=q \circ \Phi$, where $\Phi$ is the relative motion that maps reference points onto spatial points currently occupied by the material. The material description $Q$ of the field is obtained from $Q=q \circ \varphi$, where $\varphi$ is the material motion. Taking the material time derivative of $q=\hat{q} \circ \Phi^{-1}$ leads to the fundamental ALE operator

$$
\dot{q}=\frac{\partial \hat{q}}{\partial t} \circ \Phi^{-1}+c \cdot \nabla q, \quad \text { with } \quad \dot{q}=\frac{\partial Q}{\partial t} \circ \varphi^{-1} \stackrel{\text { def }}{=} h(\ldots) .
$$

The first term on the right side of the first equation represents the time derivative of $q$ with respect to fixed reference points. The second term, called the convective term, stems from the relative motion between the material and the reference domain and involves the so-called convective velocity $\boldsymbol{c}$. Finally, $h(\ldots)$ is a source or an evolution equation for the field $q$ under consideration.

The Lagrangian and Eulerian formulations are only two special cases of the ALE formulation. In the Eulerian formulation, $\boldsymbol{c}=\boldsymbol{v}=\partial \varphi / \partial t$ represents the material velocity, and $\Phi=\mathrm{id}$, for which (1) reduces to the common material time derivative. On the other hand, if the motion of the reference domain coincides with that of the material (i.e. $\boldsymbol{c}=\mathbf{0}$ and $\Phi=\varphi$ ), then the Lagrangian formulation is obtained. However, in the present work we are concerned with formulations which are essentially non-Lagrangian (Fig. 2).

Advection Algorithms The change from a Lagrangian to a non-Lagrangian viewpoint introduces two main difficulties: the presence of convective terms in the time derivatives and the problem of tracking material interfaces. The first difficulty is usually resolved either by approximating the convective terms directly, or by using conservative advection algorithms from computational fluid 


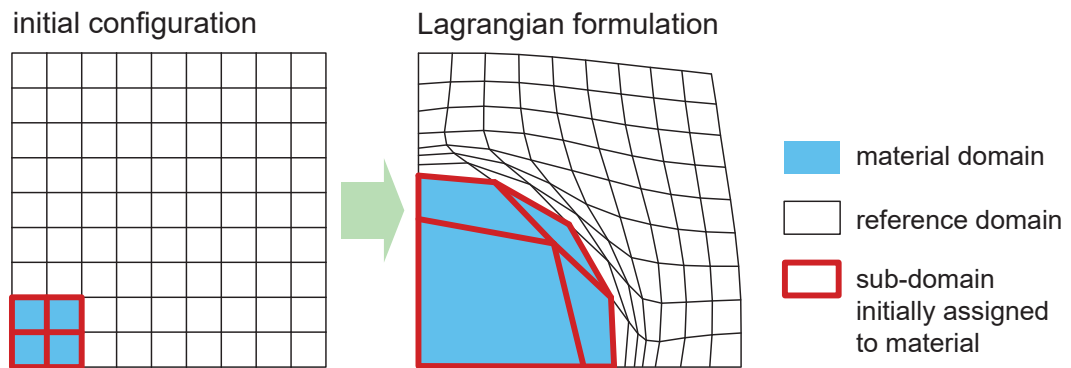

non-Lagrangian formulations

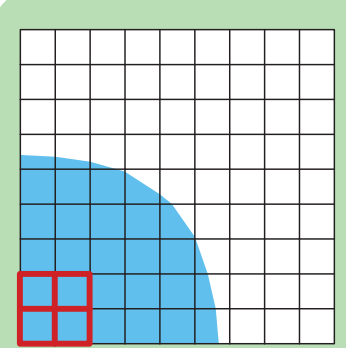

Eulerian

(multi-material case)

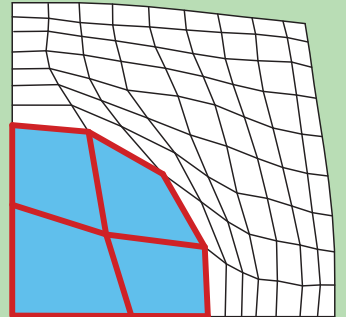

simplified ALE

(SALE)

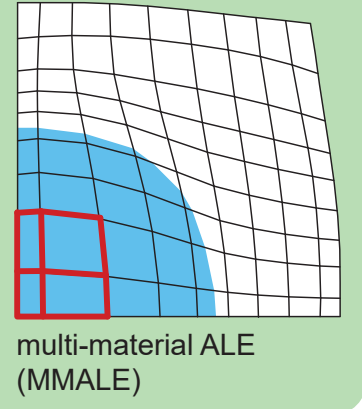

(MMALE)

Fig. 2. Schematic of Lagrangian and non-Lagrangian formulations. 
dynamics (CFD) [80,98]. However, in contrast to ideal or Newtonian fluids the constitutive behavior of soils and other geomaterials is generally path-dependent. Moreover, soil strength as well as geomorphological flows are driven by gravity and friction, which introduces additional source terms in the balance of momentum. One standard approach for solving such problems is to use the operatorsplitting resp. fractional-step technique $[33,34,52,98]$. If an operator-split is applied to the governing equations of the problems under consideration, the convective terms and the source terms are treated in separate equations which are solved sequentially.

Interface Tracking The second difficulty of tracking interfaces arises because material boundaries (free surfaces or contact interfaces) generally are not aligned with the underlying computational mesh, as they would be in a Lagrangian formulation. Since the mesh motion in ALE methods is arbitrary, it can be defined in such a way that material boundaries are resolved by edges $(2 \mathrm{~d})$ or faces (3d) of the mesh elements, and elements contain only a single material. This is called a simplified ALE (SALE) formulation $[33,34,110]$. The drawback of an SALE formulation is that the range of problems that may be addressed is not much greater than for a pure Lagrangian method because the material boundaries remain Lagrangian in both cases (Fig. 2). Methods that do not share this limitation require techniques for interface tracking $[37,88,142]$.

Interface tracking methods either track the surface, defined by a distance function or parameter representation, or the volume occupied by the material behind the interface. When using volume tracking, the material boundary is reconstructed $a b$ initio from the solution data in each mesh element containing two or more materials (so-called multi-material elements). This can be done by employing the densities of Lagrangian marker points, with the disadvantages outlined above, or alternatively, the fractional volume of each material as in volume of fluid (VOF) $[59,81,137,138,175]$ or moment of fluid (MOF) methods $[65,66]$. ALE formulations using these methods for interface tracking are referred to as full or multi-material ALE (MMALE) formulations [34,110] (Fig. 2).

Application to Geotechnical and Geomechanical Processes Concerning the modeling of geotechnical and geomechanical processes, several nonLagrangian approaches are documented in the literature. These may be classified into SALE formulations using direct approximation of the convective terms $[121,122,146]$, SALE formulations using first-order [14,16,19,20,61] and secondorder advection algorithms $[102,151]$, and Eulerian formulations using advection algorithms. The order of the advection algorithm refers to the maximum accuracy with which the spatial distribution of the solution variable is approximated. Within the Eulerian approaches, one may distinguish between channel or depthintegrated hydraulic models [60,132,134], two- or three-dimensional full-scale models using free surface tracking $[107,108]$, and multi-material full-scale models using volume tracking by VOF methods $[1,12,78]$. 
The models for the soil or debris material employed in these approaches are based on single- or two-phase descriptions ranging between simple rheological models in case of the full-scale Eulerian formulations, plastic or viscoplastic constitutive equations using the Mohr-Coulomb criterion in case of the hydraulic Eulerian formulations, and more or less advanced soil mechanical models in case of the SALE formulations. Yet no full-scale multi-material Eulerian or MMALE formulation is available which models both the complex rate-independent frictional granular material behavior and the multi-phase behavior of the grain-fluid mixture.

\subsection{Multi-Material Eulerian and MMALE Methods}

Multi-material Eulerian and MMALE (Fig. 2) are non-Lagrangian formulations that emerged along with those computational continuum mechanics tools commonly referred to as "hydrocodes" [34,110]. Typical applications include detonation and impact problems, the dynamics of bubbles and droplets, material processing and manufacturing, or astrophysical events. Besides research codes $[35,38,69,70]$ and codes developed at national laboratories for energy and defense applications $[3,59,71,106,112,115,129,165,175]$, some general purpose commercial codes include multi-material Eulerian or MMALE capabilities as well $[58,101]$; note that the fixed mesh in coupled Eulerian-Lagrangian (CEL) formulations $[40,126,135,154]$ is in fact multi-material Eulerian. The decisive advantage of an ALE mesh is that the mesh motion can be kept as Lagrangian as possible, resulting in less numerical diffusion and more accurate representation of interfaces compared to a fixed Eulerian mesh.

Three Step Scheme Implementations commonly use a Lagrange-plus-remap or three step scheme which falls into the category of operator-splitting techniques (see above). The three step scheme divides the incremental solution of the nonlinear problem into a Lagrangian step, a rezone step, and remap step (Fig. 3). During the Lagrangian step, the set of equations is solved by accounting for the sources but neglecting the convective terms; cf. (1). The rezone step relocates the nodes either to their original positions (Eulerian limit) or in such a way that mesh distortion is reduced. The remap step finally transfers the solution variables onto the modified mesh by using conservative advection algorithms. Physical time is advanced only during the Lagrangian step, whereas the spatial distributions of the solution variables are fixed during the remap step.

Subcell Closure Models (Mixture Models) The rezone step may give rise to elements which intersect with material interfaces and thus contain a heterogeneous mixture of two or more materials (Fig. 3 right). Because the spatial distribution of the element's degrees of freedom is homogeneous, however, a lack of information arises within such multi-material elements. The main difficulties are to accurately determine the states of the individual material portions and 


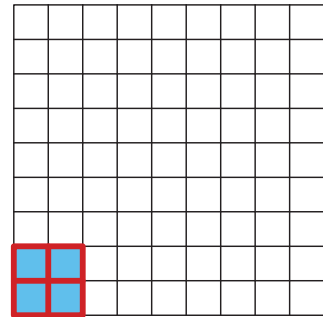

initial configuration

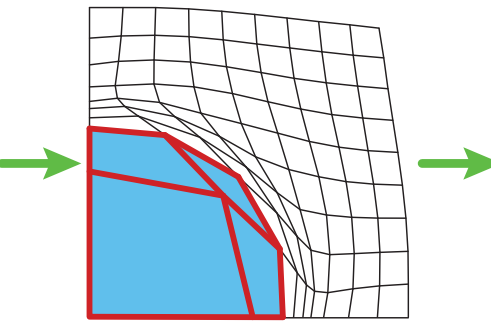

after the Lagrangian step

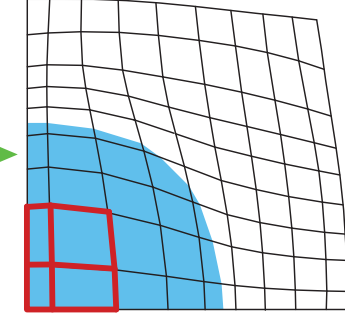

after the remap step

Fig. 3. Illustration of the MMALE three step scheme (rezone step not shown); in the multi-material Eulerian limit the rezoned mesh would be identical to the original mesh. The blue area indicates a material zone whose initial configuration is assigned to an element patch highlighted in red. At the end of a calculational cycle several elements intersect with the interface between blue and white, thus contain a mixture of two materials.

the reaction of the element they will generate [143]. To tackle these difficulties, the heterogeneous mixture is represented as an effective single-phase material (homogenized mixture). This should be based on reasonable, physically-based mixing rules, referred to as subcell closure models, which can be derived from theories incorporating material volume fraction information [36,51,53, 116,117]. However, all available subcell models assume pure, i.e. single-phase homogeneous materials at the outset, thus are not capable of including two-phase coupled response among the different materials of the mixture.

\subsection{Large-Scale Modeling of Multi-Phase Mixtures}

From the previous discussion it can be concluded that adequate non-Lagrangian models for geotechnical or geomechanical processes must account for the dynamical interaction of multiple materials on at least three different length scales $[17,21,22]$ : the scale $l_{\text {micro }}$ defined by a typical grain diameter of the granular material (microscale), the scale $l_{\text {meso }}$ at which the granular material can be represented as a continuum interacting with other bulk materials (mesoscale), and the scale $l_{\text {macro }}$ at which the immiscible mixture of mesoscale continua can be represented as an effective single-phase material (macroscale). The mesoscale is the scale commonly used in soil mechanics, and at which continuum mechanical material models operate, e.g. to reproduce the nonlinear coupled behavior of fluid-saturated sand. The solid grains and the interstitial fluid of the granular material cannot be individually distinguished. The mesoscale also carries the information of interest associated with bulk material deformation and interface evolution. On the other hand, the macoscale is typically defined by a characteristic element length in multi-material Eulerian and MMALE calculations, thus is closely connected the non-Lagrangian formulation.

Our objective is not to describe small-scale details in the multi-phase flow field, but rather large-scale motions and interactions of the materials. Flow de- 
tails should only be resolved to the extend they effect the mean flow. Upscaling information from lower to higher scales can be achieved by different types of approaches, and each has its advantages and disadvantages [26, 56, 77]. In both mathematical homogenization $[10,67,83]$ and volume averaging in the sense of Whitaker [131, 166-169], the balance equations for mass, momentum, etc., as well as the closure relations (e.g. constitutive equations) are postulated to hold on the small scale. Filtering techniques are then applied to obtain corresponding equations on the large scale at which measurements are often made. On the other hand, the continuum theory of mixtures [46-48,158] makes no small scale assumptions. Instead the system is viewed as overlapping continua with all balance principles postulated on the large scale. The form of the constitutive equations in terms of large scale variables is usually restricted by exploiting the entropy inequality.

A third type of approaches combining principles of the other two is adopted in the present research. Hybrid mixture theory was introduced in [74-77] and has been extended by Cushman and co-workers regarding two [2] and three spatial scales $[30,31,119,120]$. The basic idea is to apply local volume averaging $[45,63,64]$ to the small scale balance equations and to make the constitutive assumptions needed for closure at the large scale with respect to which averaging is carried out, that is, for the averaged balance equations. The closure relations can be obtained either by direct postulation of desirable equations, as done in [99] and herein, or based on thermodynamical considerations as in the continuum theory of mixtures.

\subsection{Overview and Structure of the Work}

This contribution summarizes the work done in Subproject 5 of the DFG Research Unit FOR 1136. The main objective of the subproject is the theoretical and numerical modeling of complex geotechnical processes such as the installation of vibro-injection piles [125,136]; cf. Fig. 1. In previous papers $[21,22,141]$, MMALE has been introduced as the authors preferred modeling framework. The main reasons for this are as follows:

- it can represent large material deformations, shear and vorticity, as well as material interface evolution including topological changes (e.g. new free surfaces are allowed to be created in a natural manner);

- it can incorporate advanced history-dependent constitutive equations and multi-phase behavior of the soil or debris material;

- it can handle interactions of multiple materials without contact elements or specific algorithms;

- it is mass conservative and can be made less numerical diffusive than pure Eulerian formulations;

- it is more versatile than Lagrangian formulations and can be applied in situations where Lagrangian formulations fail.

In accordance with the individual tasks performed to reach the objectives, the paper is structured as follows. Section 2 addresses the continuum mechanical 
modeling of saturated sand as a compressible grain-fluid mixture by starting with the most general, averaged equations for two-phase media. In Section 3, a rigorous theoretical framework is developed based upon the interpretation of geotechnical and geomechanical processes as complex multi-phase flows. This framework allows for the consideration of two-phase coupled behavior of saturated sand as well as for the construction of models for the time evolution of the material volume fractions and averaged (homogenized) properties in multi-material flow situations. The numerical techniques implemented in the Lagrangian step, the rezone step, and the remap of the developed MMALE method are outlined in Section 4. In this method, the homogeneous equilibrium model derived from the theoretical framework provides a closed set of equations that holds at each spatial point and at all interfaces (i.e. in single-material as well as in multi-material elements). Section 5 then summarizes the experimental model tests concerned with the vibro-injection pile installation. The main purposes of these tests are the verification of the assumptions underlying the theoretical investigations and the validation of the MMALE computational models. Conclusions and outlook of future work are discussed in Section 6.

\section{Saturated Sand as a Grain-Fluid Mixture}

\subsection{Averaged Equations of General Two-Phase Flow}

In this section we derive a mathematical model for grain-fluid mixtures with compressible constituents. The starting point are the most general, averaged equations describing conservation of mass and balance of linear momentum, respectively, of non-reacting immiscible two-phase flow $[63,64,89]$ :

$$
\begin{aligned}
\frac{\partial \pi^{\alpha} \rho^{\alpha}}{\partial t}+\operatorname{div}\left(\pi^{\alpha} \rho^{\alpha} \boldsymbol{v}^{\alpha}\right) & =0 \\
\frac{\partial \pi^{\alpha} \rho^{\alpha} \boldsymbol{v}^{\alpha}}{\partial t}+\operatorname{div}\left(\pi^{\alpha} \rho^{\alpha} \boldsymbol{v}^{\alpha} \otimes \boldsymbol{v}^{\alpha}\right) & =\pi^{\alpha} \rho^{\alpha} \boldsymbol{b}^{\alpha}+\operatorname{div}\left(\pi^{\alpha} \boldsymbol{\sigma}^{\alpha}\right)+\boldsymbol{\Gamma}^{\alpha} .
\end{aligned}
$$

The equations are in Eulerian conservation form, thus referring to a fixed modeling domain $\mathcal{D} \subset \mathbb{R}^{3}$ of the three-dimensional ambient Euclidian space. Each term is generally a function of point $\boldsymbol{x} \in \mathcal{D}$ and time $t \in[0, T] \subset \mathbb{R}$. The superscript $\alpha \in\{\mathrm{s}, \mathrm{f}\}$ indicates the phase, which is either solid or fluid (liquid or gas), and $\pi^{\alpha}$ is the $\alpha$-phase volume fraction with properties

$$
\pi^{\alpha} \in[0,1], \text { for all } \alpha, \quad \text { and } \quad \sum_{\alpha} \pi^{\alpha}=1 .
$$

Moreover, $\boldsymbol{v}^{\alpha}$ is the spatial image of the phase $\alpha$ material velocity, $\rho^{\alpha}$ is the spatial mass density of that phase, $\boldsymbol{b}^{\alpha}$ is a prescribed body force per unit mass, and $\boldsymbol{\sigma}^{\alpha}=\left(\boldsymbol{\sigma}^{\alpha}\right)^{\mathrm{T}}$ is the symmetric Cauchy stress. A superscribed $\mathrm{T}$ denotes transposition of a second-order tensor, $\otimes$ is the tensor product, and div is the spatial divergence operator. The momentum interfacial transfer term $\Gamma^{\alpha}$ includes surface drag forces per unit volume generated by the relative motion 
of the phases. Here we simply assume that both phases move with the same velocity, i.e. $\boldsymbol{v}^{\mathrm{s}}=\boldsymbol{v}^{\mathrm{f}}$, resulting in $\boldsymbol{\Gamma}^{\mathrm{s}}=-\boldsymbol{\Gamma}^{\mathrm{f}} \equiv \mathbf{0}$. In fluid-saturated granular material this formalizes locally undrained conditions $[99,176]$.

The stress tensor is usually decomposed into a pressure stress and an extra stress according to $[111,159]$

$$
\boldsymbol{\sigma}^{\alpha}=-p^{\alpha} \boldsymbol{I}+\boldsymbol{s}^{\alpha}
$$

where $\boldsymbol{I}$ is the second-order unit tensor. In general, the changes in pressure stress are related to changes in mass density, whereas the extra stress is related to material deformations. For simplicity, we let $p^{\alpha}=-\frac{1}{3} \operatorname{tr} \boldsymbol{\sigma}^{\alpha}$, meaning that the extra stress is deviatoric, i.e. $s^{\alpha}=\sigma_{\mathrm{dev}}^{\alpha}$, where $\operatorname{tr}$ denotes the trace of a second-order tensor and $\boldsymbol{\sigma}_{\mathrm{dev}} \stackrel{\text { def }}{=} \boldsymbol{\sigma}-\frac{1}{3}(\operatorname{tr} \boldsymbol{\sigma}) \boldsymbol{I}$.

We remark that quantities in (2) and (3) have been averaged with respect to a representative volume element (RVE). Because of $(4)_{2}$, summation over both phases yields the mixture balance equations

$$
\begin{aligned}
\frac{\partial \bar{\rho}}{\partial t}+\operatorname{div}(\bar{\rho} \overline{\boldsymbol{v}}) & =0, \\
\frac{\partial \bar{\rho} \overline{\boldsymbol{v}}}{\partial t}+\operatorname{div}(\bar{\rho} \overline{\boldsymbol{v}} \otimes \overline{\boldsymbol{v}}) & =\bar{\rho} \overline{\boldsymbol{b}}+\operatorname{div} \overline{\boldsymbol{\sigma}},
\end{aligned}
$$

respectively. For example, $\bar{\rho}=\sum_{\alpha} \pi^{\alpha} \rho^{\alpha}$.

Provided that the $\boldsymbol{\sigma}^{\alpha}$ have already been modeled by appropriate constitutive equations, the set (2) and (3) constitutes a system of $2 \cdot 2$ equations in the $3 \cdot 2-1$ unknowns $\pi^{\alpha}, \rho^{\alpha}$, and $\boldsymbol{v}^{\alpha}$, with $\alpha \in\{\mathrm{s}, \mathrm{f}\}$, where we emphasize that the $\pi^{\alpha}$ should only be considered and counted as only one variable because of $(4)_{2}$. Therefore, the system of equation requires one additional relation for the volume fraction, called the topological closure relation $[44,45,51]$, to close the system. Otherwise the system would be indeterminate.

\subsection{Stress Contributions in Granular Material}

We consider a cohesionless granular material in which a single fluid fills the interstitial space. Rheologists call this a dense grain-fluid mixture or granular suspension [5], and a common example is saturated sand. Since only two phases are present, we simply define the fluid fraction or porosity through

$$
n \stackrel{\text { def }}{=} \pi^{\mathrm{f}}, \quad \text { so that } \quad \pi^{\mathrm{s}}=1-n
$$

by using $(4)_{2}$.

Two limiting regimes of dry granular flow are usually considered $[9,86,139]$ : a rate-independent frictional flow regime usually studied in soil mechanics [144, $176]$, and a rate-dependent viscous flow regime where grain inertia and instantaneous grain contacts through collision dominate $[23,86,87]$. In the intermediate, frictional-collisional flow regime, the contributions of frictional and collisional interactions to the bulk stress of the mixture cannot be clearly distinguished $[6-8,93]$. 
Further complexity is introduced by the interstitial fluid in granular materials. Besides the consolidation and liquefaction phenomena well-known in soil mechanics, indirect grain interactions may occur through lubricated contacts $[5,6,8,54]$. Generally all flow regimes have to be considered in the analysis of geotechnical and geomechanical processes. However, yet no constitutive equation is available which models the mechanical behavior of dense grain-fluid mixture over a wide range of flow conditions and material properties [8].

To account for the different flow regimes, the stress tensors of the solid and fluid phases in grain-fluid mixtures are additively decomposed into a rateindependent frictional part and a rate-dependent viscous part $[9,87,93]$ :

$$
\boldsymbol{\sigma}^{\alpha} \stackrel{\text { def }}{=} \boldsymbol{\sigma}_{\mathrm{fr}}^{\alpha}+\boldsymbol{\sigma}_{\mathrm{vi}}^{\alpha}, \quad \text { with } \quad \alpha \in\{\mathrm{s}, \mathrm{f}\}
$$

Terzaghi's effective stress [153], $\boldsymbol{\sigma}_{\mathrm{fr}}^{\prime}$, is introduced as

$$
\frac{\boldsymbol{\sigma}_{\mathrm{fr}}^{\prime}}{1-n} \stackrel{\text { def }}{=}-\left(p^{\mathrm{s}}-p^{\mathrm{f}}\right) \boldsymbol{I}+\boldsymbol{s}_{\mathrm{fr}}^{\mathrm{s}}
$$

in accordance with [32], so that

$$
\frac{p^{\prime}}{1-n}=p^{\mathrm{s}}-p^{\mathrm{f}}
$$

where $p^{\prime} \stackrel{\text { def }}{=}-\frac{1}{3} \operatorname{tr} \boldsymbol{\sigma}_{\mathrm{fr}}^{\prime}$ is the (negative) mean effective stress and $p^{\mathrm{s}}-p^{\mathrm{f}}$ is the excess pressure.

A particular form of the principle of effective stress $[144,176]$ can be derived by combining (10) with an expression of the total stress of the grain-fluid mixture,

$$
\overline{\boldsymbol{\sigma}}=(1-n) \boldsymbol{\sigma}^{\mathrm{s}}+n \boldsymbol{\sigma}^{\mathrm{f}}
$$

resulting in

$$
\overline{\boldsymbol{\sigma}}_{\mathrm{fr}}=\boldsymbol{\sigma}_{\mathrm{fr}}^{\prime}-p^{\mathrm{f}} \boldsymbol{I} .
$$

We adopt this form for conceptual reasons, while noting that several other versions have been postulated; cf. [96].

If the effective stress for the viscous part is assumed unaffected by fluid stresses, then substitution of (13) and (9) into (12) yields the following representation of the principle of effective stress for a general grain-fluid mixture [92]:

$$
\overline{\boldsymbol{\sigma}}=\boldsymbol{\sigma}_{\mathrm{fr}}^{\prime}+\boldsymbol{\sigma}_{\mathrm{vi}}^{\prime}+\boldsymbol{\sigma}_{\mathrm{fr}}^{\mathrm{f}}+n \boldsymbol{\sigma}_{\mathrm{vi}}^{\mathrm{f}}=\boldsymbol{\sigma}^{\prime}-p^{\mathrm{f}} \boldsymbol{I}+n \boldsymbol{s}^{\mathrm{f}},
$$

with $\boldsymbol{\sigma}^{\prime}=\boldsymbol{\sigma}_{\mathrm{fr}}^{\prime}+\boldsymbol{\sigma}_{\mathrm{vi}}^{\prime}$. In terms of pressure stress, the principle (14) reads $\bar{p}=p^{\prime}+p^{\mathrm{f}}$, where $\bar{p} \stackrel{\text { def }}{=}-\frac{1}{3} \operatorname{tr} \overline{\boldsymbol{\sigma}}_{\mathrm{fr}}$.

\subsection{Constitutive Equations}

Application of (14) requires models for $\boldsymbol{\sigma}_{\mathrm{fr}}^{\prime}, \boldsymbol{\sigma}_{\mathrm{vi}}^{\prime}, p^{\mathrm{f}}$, and $\boldsymbol{s}^{\mathrm{f}}$. The fluid phase is usually represented as a Newtonian fluid, leading to simple representations of 
$p^{\mathrm{f}}$ and $\boldsymbol{s}^{\mathrm{f}}$. Concerning the frictional part of effective stress, $\boldsymbol{\sigma}_{\mathrm{fr}}^{\prime}$, a large number of constitutive equations has been proposed for applications in soil mechanics. States of failure can be adequately modeled by models employing the classical Mohr-Coulomb yield condition [176]. Comprehensive constitutive equations that might be applied beyond states of failure fall into the categories of hypoelastoplastic $[100,113,128,152]$ or hypoplastic $[73,95,123,164]$ rate constitutive equations. Significant progress in the development of hypoplasticity has also been achieved in the context of this DFG Research Unit [104,124]. Such equations take the general form

$$
\stackrel{\nabla}{\mathrm{fr}}_{\mathrm{fr}} \stackrel{\text { def }}{=} \boldsymbol{c}_{\mathrm{fr}}^{\prime}\left(\boldsymbol{\sigma}_{\mathrm{fr}}^{\prime}, n, \boldsymbol{h}\right): \boldsymbol{d},
$$

by assuming incompressible constituents. Here $\stackrel{\nabla}{\sigma}$ denotes any objective rate of $\boldsymbol{\sigma}, \boldsymbol{c}$ is the fourth-order material tangent tensor, $\boldsymbol{h}$ is a set of history variables other than stress, and $\boldsymbol{d} \stackrel{\text { def }}{=} \frac{1}{2}\left(\boldsymbol{\nabla} \boldsymbol{v}+(\boldsymbol{\nabla} \boldsymbol{v})^{\mathrm{T}}\right)$ is the spatial rate of deformation tensor.

Constitutive equations for the viscous part of the effective stress, $\boldsymbol{\sigma}_{\mathrm{vi}}^{\prime}$, are often restricted to particular flow conditions or to narrow ranges of material properties. Common models take the form $[87,127]$

$$
\boldsymbol{\sigma}_{\mathrm{vi}}^{\prime} \stackrel{\text { def }}{=} \mu^{\prime} \boldsymbol{d}_{\mathrm{dev}}^{\mathrm{s}}
$$

where $\mu^{\prime}$ is the dynamic shear viscosity. The latter is generally a function of the porosity resp. solid volume fraction and shear rate $[23,68,86,87,93,94,127,130]$.

\subsection{Topological Closure and Compressible Constituents}

Sect. 2.1 has revealed that a well-posed model for grain-fluid mixtures requires topological closure, that is, an evolution equation for the fluid volume fraction (porosity). If the material of one phase, say, the solid phase is incompressible, then this missing equation is readily obtained from conservation of mass of that phase. However, an indeterminacy arises for compressible constituents, reflected in the fact that $\pi^{\alpha}$ and $\rho^{\alpha}$ appear in the general equations (2) and (3) only in the form of the product $\tilde{\rho}^{\alpha}=\pi^{\alpha} \rho^{\alpha}$, but not separately.

A Lagrangian formulation is chosen to resolve the aforementioned indeterminacy. First, let the material time derivative of a $\alpha$-phase-related quantity $q^{\alpha}$ along the velocity $\boldsymbol{v}^{\alpha}$ be defined through

$$
\dot{q}^{\alpha} \stackrel{\text { def }}{=} \frac{\partial q^{\alpha}}{\partial t}+\boldsymbol{v}^{\alpha} \cdot \nabla q^{\alpha}, \quad \text { with } \quad \alpha \in\{\mathrm{s}, \mathrm{f}\},
$$

where $\boldsymbol{\nabla}$ is the covariant derivative, and $\boldsymbol{v}^{\mathrm{s}}=\boldsymbol{v}^{\mathrm{f}}=\overline{\boldsymbol{v}}$, i.e. locally undrained conditions have been assumed. Conservation of mass (2) for the solid phase can then be written in Lagrangian form:

$$
\frac{\dot{\tilde{\rho}}^{\mathrm{s}}}{\tilde{\rho}^{\mathrm{s}}}=-\operatorname{div} \boldsymbol{v}^{\mathrm{s}} .
$$

with the bulk mass density $\tilde{\rho}^{\mathrm{s}}=\pi^{\mathrm{s}} \rho^{\mathrm{s}}=(1-n) \rho^{\mathrm{s}}$ of the solid phase. 
The decomposition of stress (5) introduces pressure as an independent variable. The pressure change of each phase is related to its change in density through a compression model of the form

$$
-\left.\frac{1}{V^{\alpha}} \frac{\partial V^{\alpha}}{\partial p^{\alpha}}\right|_{M^{\alpha}}=\frac{1}{\rho^{\alpha}} \frac{\mathrm{d} \rho^{\alpha}}{\mathrm{d} p^{\alpha}} \stackrel{\text { def }}{=} \frac{1}{K^{\alpha}}, \quad \text { with } \quad \alpha \in\{\mathrm{s}, \mathrm{f}\} .
$$

$K^{\alpha}$ is the bulk modulus of the $\alpha$-phase material, $V^{\alpha}=\pi^{\alpha} V$ is the volume occupied by phase $\alpha$ within a small Lagrangian control volume $V$ of the mixture, $M^{\alpha}=\rho^{\alpha} V^{\alpha}$ is the mass of that phase volume, and $\left.\right|_{M^{\alpha}}$ means that the mass of the $\alpha$-phase is kept constant along with differentiation.

In a mixture the pressure of each compressible constituent is generally a function of the mass density and volume fraction of that constituent. In particular, the pressure of the solid phase (grains) in granular material does not only depend on the mass density but also on the porosity [28, 29]. However, one usually assumes that the Lagrangian control volume occupied by the grain-fluid mixture is a function

$$
V=V\left(p^{\mathrm{f}}, \bar{p}-p^{\mathrm{f}}\right)
$$

of the fluid phase pressure and the pressure difference $\bar{p}-p^{\mathrm{f}}=p^{\prime}$. Under the assumption that total mass of the solid phase, $M^{\mathrm{s}}$, is kept fixed, one has

$$
\begin{aligned}
\left.\frac{\mathrm{d} V}{V}\right|_{M^{\mathrm{s}}} & =\left.\frac{1}{V} \frac{\partial V}{\partial\left(\bar{p}-p^{\mathrm{f}}\right)}\right|_{p^{\mathrm{f}}, M^{\mathrm{s}}} \mathrm{d}\left(\bar{p}-p^{\mathrm{f}}\right)+\left.\frac{1}{V} \frac{\partial V}{\partial p^{\mathrm{f}}}\right|_{\bar{p}-p^{\mathrm{f}}, M^{\mathrm{s}}} \mathrm{d} p^{\mathrm{f}} \\
& =\left.\frac{1}{V} \frac{\partial V}{\partial \bar{p}}\right|_{p^{\mathrm{f}}, M^{\mathrm{s}}} \mathrm{d}\left(\bar{p}-p^{\mathrm{f}}\right)+\left.\frac{1}{V} \frac{\partial V}{\partial p^{\mathrm{f}}}\right|_{\bar{p}-p^{\mathrm{f}}, M^{\mathrm{s}}} \mathrm{d} p^{\mathrm{f}} \\
& \stackrel{\text { def }}{=}-\frac{1}{K_{\mathrm{dr}}} \mathrm{d}\left(\bar{p}-p^{\mathrm{f}}\right)-\frac{1}{K_{\mathrm{uj}}} \mathrm{d} p^{\mathrm{f}}
\end{aligned}
$$

where $K_{\mathrm{dr}}$ and $K_{\mathrm{uj}}$ are called the drained bulk modulus and unjacketed bulk modulus of the granular material, respectively, and

$$
K_{\mathrm{uj}} \approx K^{\mathrm{s}}
$$

By recalling that $M^{\mathrm{s}}=\rho^{\mathrm{s}} V^{\mathrm{s}}=\rho^{\mathrm{s}}(1-n) V$, Eq. (18) is equivalent to

$$
\frac{\mathrm{d} \tilde{\rho}^{\mathrm{s}}}{\tilde{\rho}^{\mathrm{s}}}=-\left.\frac{\mathrm{d} V}{V}\right|_{M^{\mathrm{s}}}
$$

Therefore, replacing in (21) the total differential with the material time derivative yields

$$
\dot{\bar{p}}=-K_{\mathrm{dr}} \operatorname{div} \boldsymbol{v}^{\mathrm{s}}+\zeta \dot{p}^{\mathrm{f}}
$$

where $\zeta \stackrel{\text { def }}{=} 1-K_{\mathrm{dr}} / K^{\mathrm{s}}$ is the Biot-Willis coefficient $[42,43,176]$.

Based on the previous results together with the definition of mean effective stress, (11), we are now able to relate solid and fluid phase pressures, $p^{\mathrm{s}}$ and $p^{\mathrm{f}}$, to solid phase volumetric deformation. To determine the rate of the solid pressure, 
we start from the relative volume change $\mathrm{d} V^{\mathrm{s}} /\left.V^{\mathrm{s}}\right|_{(1-n)}$ by keeping solid phase volume fraction constant:

$$
\begin{aligned}
\left.\frac{\mathrm{d} V^{\mathrm{s}}}{V^{\mathrm{s}}}\right|_{1-n} & =-\frac{1}{\rho^{\mathrm{s}}} \frac{\partial \rho^{\mathrm{s}}}{\partial p^{\mathrm{s}}} \mathrm{d} p^{\mathrm{s}}=-\frac{\mathrm{d} p^{\mathrm{s}}}{K^{\mathrm{s}}}=-\frac{1}{K^{\mathrm{s}}}\left(\frac{\mathrm{d} p^{\prime}}{1-n}+\mathrm{d} p^{\mathrm{f}}\right) \\
& =\left(\left.\frac{\mathrm{d} V}{V}\right|_{M^{\mathrm{s}}}+\frac{\mathrm{d} p^{\mathrm{f}}}{K^{\mathrm{s}}}\right) \frac{K_{\mathrm{dr}}}{(1-n) K^{\mathrm{s}}}-\frac{\mathrm{d} p^{\mathrm{f}}}{K^{\mathrm{s}}} \\
& =\left.\frac{K_{\mathrm{dr}}}{(1-n) K^{\mathrm{s}}} \frac{\mathrm{d} V}{V}\right|_{M^{\mathrm{s}}}-\left(1-\frac{K_{\mathrm{dr}}}{(1-n) K^{\mathrm{s}}}\right) \frac{\mathrm{d} p^{\mathrm{f}}}{K^{\mathrm{s}}},
\end{aligned}
$$

By replacing the total differential with the material time derivative again and rearrange, one obtains

$$
\dot{p}^{\mathrm{s}}=-\frac{K_{\mathrm{dr}}}{1-n} \operatorname{div} \boldsymbol{v}^{\mathrm{s}}+\frac{\zeta-n}{1-n} \dot{p}^{\mathrm{f}} .
$$

On the other hand, the assumption of locally undrained conditions, i.e. $\boldsymbol{v}^{\mathrm{f}}=$ $\boldsymbol{v}^{\mathrm{s}}=\overline{\boldsymbol{v}}$, allows us to rewrite conservation of mass (2) for the fluid phase as

$$
\dot{p}^{\mathrm{f}}=-\zeta Q \operatorname{div} \boldsymbol{v}^{\mathrm{s}}, \quad \text { with } \quad Q \stackrel{\text { def }}{=}\left(\frac{\zeta-n}{K^{\mathrm{s}}}+\frac{n}{K^{\mathrm{f}}}\right)^{-1} .
$$

Substitution of (27) into (24) finally yields a Lagrangian form of (6):

$$
\dot{\bar{p}}=-K \operatorname{div} \overline{\boldsymbol{v}}
$$

in which

$$
K=K_{\mathrm{dr}}\left(1+\frac{\zeta^{2}}{\zeta \frac{K_{\mathrm{dr}}}{K^{\mathrm{s}}}+n\left(\frac{K_{\mathrm{dr}}}{K^{\mathrm{f}}}-\frac{K_{\mathrm{dr}}}{K^{\mathrm{s}}}\right)}\right) .
$$

Eq. (28) in conjunction with the bulk modulus given by (29) holds for arbitrary compositions of saturated grain-fluid mixtures with compressible constituents and homogeneous velocity, that is, undrained conditions. Particular cases included are:

- solid without any pores $\left(n=0, K_{\mathrm{dr}}=K^{\mathrm{s}}, \zeta=0\right)$, for which $K=K^{\mathrm{s}}$;

- fluid without any solid content $\left(n=1, K_{\mathrm{dr}}=0, \zeta=1\right)$, for which $K=K^{\mathrm{f}}$;

- dry granular material $\left(0<n<1, K^{\mathrm{f}} \approx 0\right)$, for which $K=K_{\mathrm{dr}}$;

- uniform suspension of zero friction $\left(K_{\mathrm{dr}}=0, \zeta=1\right)$, for which $p^{\mathrm{s}}=p^{\mathrm{f}}$ and $K=\left((1-n) / K^{\mathrm{s}}+n / K^{\mathrm{f}}\right)^{-1}$, known as Wood's equation [172, p. 327].

In concluding this section, we remark that the definition (11) of mean effective stress resolves the indeterminacy associated with volume fraction evolution in compressible grain-fluid mixtures. This definition provides the missing link 
between volumetric deformation and changes in solid and fluid pressures. Evolution of volume fraction in two-phase grain-fluid mixtures thus can be associated with solid phase conservation of mass (2) resp. (18) alone:

$$
\dot{n}=(1-n)\left(\frac{\dot{p}^{\mathrm{s}}}{K^{\mathrm{s}}}+\operatorname{div} \boldsymbol{v}^{\mathrm{s}}\right)
$$

in which $\dot{p}^{\text {s }}$ is given by $(26)$.

\section{Mixture Model for Multi-Material Interaction}

The previous section was concerned with the continuum mechanical modeling of general two-phase, grain-fluid mixtures, of which saturated sand is but one example. It has been shown that if both compressible phases move with the same velocity, representing locally undrained conditions, then the mixture can be equivalently modeled as an effective single-phase, i.e. homogeneous bulk material. In the section that follows, we summarize a rigorous theoretical framework we have developed $[17,21,22]$ to construct macroscopic mixture models for the dynamical interaction of grain-fluid mixtures with multiple other, physically distinct bulk materials, e.g. pure fluids or pure solids. Void, representing empty space or atmosphere, is generally considered as material, and all materials may undergo large deformations. We refer to such a situation as multi-material flow.

\subsection{Averaging Procedure}

Consider a three-scale system consisting of bulk fluid (F), bulk solid (S), and fluid-saturated granular material (G). The granular material consists of a solid phase (s) and fluid phase (f), and is constituted by an assembly of solid grains, whose typical diameter defines the microscale of the problem, $l_{\text {micro }}$. The grain assembly can be represented by a continuum at the mesoscale $l_{\text {meso }}$, separated from the bulk solid and bulk fluid by sharp interfaces. Moreover, we assume that the multi-material system has a representative volume element (RVE) with characteristic length $l_{\text {macro }}$, the macroscale. The RVE is a sub-domain $\mathcal{H} \subset \mathcal{D}$ of the spatially fixed, i.e. Eulerian modeling domain introduced in Sect. 2.1.

Let $k \in\{\mathrm{S}, \mathrm{F}, \mathrm{G}\} \stackrel{\text { def }}{=}\{1, \ldots, M\}$ denote the material and $\alpha \in\{\mathrm{s}, \mathrm{f}\} \stackrel{\text { def }}{=}$ $\{1, \ldots, N\}$ the phase. A particular phase $\alpha$ in a particular material $k$ represents an individual, chemically-independent constituent of the flow and is denoted by $\alpha k$. Concerning the present situation, $\alpha k \in\{\mathrm{S} \equiv \mathrm{sS}, \mathrm{F} \equiv \mathrm{fF}, \mathrm{sG}, \mathrm{fG}\}$. The intersection of each two constituents is either empty or the shared interface. The indicator function $\chi^{\alpha k}: \mathcal{D} \times[0, T] \rightarrow\{0,1\}$ which picks out the $\alpha$-phase domain of the $k$-material domain is defined by

$$
\chi^{\alpha k}(\boldsymbol{x}, t) \stackrel{\text { def }}{=} \begin{cases}1 & \text { if } \boldsymbol{x} \text { is in } \alpha k \text { at time } t \\ 0 & \text { else. }\end{cases}
$$


The point we wish to emphasize is that the indicator function (31) can be represented as the product $\chi^{\alpha k}=\chi^{\alpha} \chi^{k}$ of two independent indicator functions for each material $k$ and each phase $\alpha$.

As introduced in Sect. 1, our approach employs hybrid mixture theory to upscale information from the microscale to the macroscale. The microscopic balance equations are upscaled by using local volume averaging, and then the closure relations (constitutive equations, etc.) are postulated on the macroscale. To this end, let the subregion of the RVE occupied by the $k$-material be $\mathcal{H}^{k}$, and let $\mathcal{H}^{\alpha k}$ be the subregion occupied by the $\alpha$-phase of the $k$-material, with $\mathcal{H}=\bigcup_{k} \mathcal{H}^{k}=\bigcup_{k} \bigcup_{\alpha} \mathcal{H}^{\alpha k}$. Then the $\mathcal{H}$-average of an arbitrary time-dependent spatial microscopic field $q(\boldsymbol{x}, t)$ is defined through

$$
\langle q\rangle(\boldsymbol{x}, t) \stackrel{\text { def }}{=} \frac{1}{H} \int_{\mathcal{H}} q(x+\boldsymbol{y}, t) \mathrm{d} v
$$

for all $\boldsymbol{x} \in \mathcal{D}$ and $t \in[0, T]$, where $\mathrm{d} v$ is the volume density on $\mathbb{R}^{3}, H \stackrel{\text { def }}{=}$ $\int_{\mathcal{H}} 1 \mathrm{~d} v=$ const is the volume measure of $\mathcal{H}$, and $\boldsymbol{y} \in \mathcal{H}$ is a vector.

Two particular averaged fields frequently used are the volume fractions

$$
f^{k} \stackrel{\text { def }}{=}\left\langle\chi^{k}\right\rangle=\frac{H^{k}}{H} \quad \text { and } \quad \pi^{\alpha k} \stackrel{\text { def }}{=} \frac{1}{f^{k}}\left\langle\chi^{\alpha k}\right\rangle=\frac{H^{\alpha k}}{H^{k}},
$$

where $H^{k} \stackrel{\text { def }}{=} \int_{\mathcal{H}^{k}} 1 \mathrm{~d} v=\int_{\mathcal{H}} \chi^{k} \mathrm{~d} v$ and $H^{\alpha k} \stackrel{\text { def }}{=} \int_{\mathcal{H}^{\alpha k}} 1 \mathrm{~d} v=\int_{\mathcal{H}} \chi^{\alpha k} \mathrm{~d} v$. While $f^{k}$ is the volume fraction of the $k$-material with respect to the RVE, $\pi^{\alpha k}$ represents the macroscale volume fraction of the $\alpha$-phase intrinsic to the $k$-material, with $f^{k}, \pi^{\alpha k} \in[0,1]$. Phase or material overlaps are not allowed, hence

$$
\sum_{k} f^{k}=1 \quad \text { and } \quad \sum_{\alpha} \pi^{\alpha k}=1
$$

for all $k \in\{1, \ldots, M\}$.

Different macroscopic (i.e. $\mathcal{H}$-averaged) fields can now be defined by employing the previous definitions and the properties (34). Clearly, if $q$ is a microscopic field defined per unit volume, then

$$
\langle q\rangle=\sum_{k} f^{k} q^{k}=\sum_{k} \sum_{\alpha} f^{k} \pi^{\alpha k} q^{\alpha k},
$$

with

$$
q^{k} \stackrel{\text { def }}{=} \frac{\left\langle\chi^{k} q\right\rangle}{f^{k}} \quad \text { and } \quad q^{\alpha k} \stackrel{\text { def }}{=} \frac{\left\langle\chi^{\alpha k} q\right\rangle}{f^{k} \pi^{\alpha k}} .
$$

Here $\langle q\rangle$ is the volume average of $q$ in the mixture, $q^{k}$ is the intrinsic $k$-material average, and $q^{\alpha k}$ is the intrinsic average related to the $\alpha$-phase in the $k$-material. Accordingly, if $q=\rho$, for example, is the microscopic spatial mass density, then $\rho^{\alpha k}$ represents the mass of the constituent $\alpha k$ per unit volume of that constituent, $\pi^{\alpha k} \rho^{\alpha k}$ is the mass of the constituent $\alpha k$ per unit volume of the $k$-material, and $f^{k} \pi^{\alpha k} \rho^{\alpha k}$ denotes its mass per unit volume of the mixture. 
We remark that, in the present approach, each material represents a mixture generally composed of a solid phase and a fluid phase. Hence, we define

$$
n^{k \stackrel{\text { def }}{=}} \pi^{\mathrm{f} k}
$$

as the fluid fraction or porosity of material $k$, with $k \in\{\mathrm{S}, \mathrm{F}, \mathrm{G}\}$; cf. (8). Then the solid fraction is $\pi^{\mathrm{s} k}=1-n^{k}$ by using $(34)_{2}$. For pure solid $(k=\mathrm{S})$ one has $n^{\mathrm{S}}=0$, whereas $n^{\mathrm{F}}=1$ in case of pure fluid $(k=\mathrm{F})$.

\subsection{Macroscopic Mixture Model}

On the microscale all constituents of the mixture are regarded as continua, governed by the equations of continuum mechanics $[111,158,159]$. The balance equations are conservation of mass and balance of momentum for the problems under consideration, in conjunction with the interface jump conditions. Each term of the microscopic balance equations is averaged according to the procedure above. Details can be found in $[21]$, and in $[63,64]$ for the case of two scales.

Under the assumption of non-reactive constituents, one obtains the following Eulerian conservation form of $\alpha$-phase- $k$-material macroscopic conservation of mass

$$
\frac{\partial f^{k} \pi^{\alpha k} \rho^{\alpha k}}{\partial t}+\operatorname{div}\left(f^{k} \pi^{\alpha k} \rho^{\alpha k} \boldsymbol{v}^{\alpha k}\right)=0
$$

and macroscopic balance of linear momentum

$$
\begin{aligned}
& \frac{\partial f^{k} \pi^{\alpha k} \rho^{\alpha k} \boldsymbol{v}^{\alpha k}}{\partial t}+\operatorname{div}\left(f^{k} \pi^{\alpha k} \rho^{\alpha k} \boldsymbol{v}^{\alpha k} \otimes \boldsymbol{v}^{\alpha k}\right)= \\
& f^{k} \pi^{\alpha k} \rho^{\alpha k} \boldsymbol{b}^{\alpha k}+\operatorname{div}\left(f^{k} \pi^{\alpha k} \boldsymbol{\sigma}^{\alpha k}\right)+\boldsymbol{\Gamma}^{\alpha k} .
\end{aligned}
$$

Note that these equations are consistent with the set (2) and (3) for general two-phase media if one sets $\chi^{k} \equiv 1$ resp. $f^{k} \equiv 1$ (single material case). As in Sect. 2.1, let us further assume that no momentum is exchanged no matter between which constituents, and that velocity is homogeneous. Clearly,

$$
\boldsymbol{\Gamma}^{\alpha k} \equiv \mathbf{0} \quad \text { and } \quad\langle\boldsymbol{v}\rangle=\boldsymbol{v}^{k}=\boldsymbol{v}^{\alpha k}
$$

for all $k \in\{\mathrm{S}, \mathrm{F}, \mathrm{G}\}$ and $\alpha k \in\{\mathrm{S}, \mathrm{F}, \mathrm{sG}, \mathrm{fG}\}$.

Based on (35), summation of (38) and (39) over all phases and all materials finally yield the macroscopic conservation of mass and macroscopic balance of momentum of the mixture:

$$
\begin{gathered}
\frac{\partial\langle\rho\rangle}{\partial t}+\operatorname{div}\langle\rho \boldsymbol{v}\rangle=0 \\
\frac{\partial\langle\rho \boldsymbol{v}\rangle}{\partial t}+\operatorname{div}\langle\rho \boldsymbol{v} \otimes \boldsymbol{v}\rangle=\langle\rho \boldsymbol{b}\rangle+\operatorname{div}\langle\boldsymbol{\sigma}\rangle .
\end{gathered}
$$

We emphasize again the consistency between (41) and(6), as well as between (42) and (7) for the case where the mixture represents a single saturated granular material and no other bulk materials. Moreover, Sect. 2 has revealed that 
grain-fluid mixtures without mass and momentum exchange can be treated as homogeneous bulk material, possessing a single velocity field. Therefore, after adding the superscript $\mathrm{G}$ referring to the granular material to the relevant terms of Sect. 2, these terms can be directly substituted into the equations of this section.

After combining the mixture balance principles (41) and (42) with the particularizations made for saturated granular material in Sect. 2, and doing some algebraic manipulation, the following Lagrangian model for geotechnical or geomechanical multi-material flow is obtained $[17,21]$ :

$$
\begin{aligned}
\langle\dot{p}\rangle /\langle K\rangle+\operatorname{div}\langle\boldsymbol{v}\rangle & =0 \\
\operatorname{div}\langle\boldsymbol{s}-p \boldsymbol{I}\rangle+\langle\rho \boldsymbol{b}\rangle-\langle\rho\rangle\langle\boldsymbol{v}\rangle & =\mathbf{0},
\end{aligned}
$$

in which

$$
\begin{aligned}
\langle p\rangle=\sum_{k} f^{k} p^{k}=f^{\mathrm{S}} p^{\mathrm{S}}+f^{\mathrm{F}} p^{\mathrm{F}}+f^{\mathrm{G}}\left(p^{\mathrm{G}^{\prime}}+p^{\mathrm{fG}}\right), \\
\langle\boldsymbol{s}\rangle=\sum_{k} f^{k} \boldsymbol{s}^{k}=f^{\mathrm{S}} \boldsymbol{s}^{\mathrm{S}}+f^{\mathrm{F}} \boldsymbol{s}^{\mathrm{F}}+f^{\mathrm{G}}\left(\boldsymbol{s}^{\mathrm{G}^{\prime}}+n^{\mathrm{G}} \boldsymbol{s}^{\mathrm{fG}}\right), \\
\langle\rho\rangle=\sum_{k} f^{k} \rho^{k} \\
=f^{\mathrm{S}} \rho^{\mathrm{S}}+f^{\mathrm{F}} \rho^{\mathrm{F}}+f^{\mathrm{G}}\left(\left(1-n^{\mathrm{G}}\right) \rho^{\mathrm{SG}}+n^{\mathrm{G}} \rho^{\mathrm{fG}}\right), \\
\frac{1}{\langle K\rangle}=\sum_{k} \frac{f^{k}}{K^{k}}=\frac{f^{\mathrm{S}}}{K^{\mathrm{S}}}+\frac{f^{\mathrm{F}}}{K^{\mathrm{F}}}+\frac{f^{\mathrm{G}}}{K^{\mathrm{G}}},
\end{aligned}
$$

and $K^{\mathrm{G}}$ given by (29).

Constitutive equations and compression models must be prescribed for the bulk solid $\left(\boldsymbol{s}^{\mathrm{S}}, p^{\mathrm{S}}\right)$, bulk fluid $\left(\boldsymbol{s}^{\mathrm{F}}, p^{\mathrm{F}}\right)$, granular material $\left(\boldsymbol{s}^{\mathrm{G}^{\prime}}, p^{\mathrm{G}^{\prime}}\right)$, and interstitial fluid $\left(s^{\mathrm{fG}}, p^{\mathrm{fG}}\right)$, by using the consistent macroscopic rate of deformation tensor

$$
\left\langle\boldsymbol{d}_{k}\right\rangle \stackrel{\text { def }}{=}\langle\boldsymbol{d}\rangle+\frac{\dot{f}^{k}}{3 f^{k}} \boldsymbol{I} \quad \text { for each } k \in\{\mathrm{S}, \mathrm{F}, \mathrm{G}\} .
$$

Moreover, in accordance with Sect. 2.4 the evolution of the porosity of the granular material is given by

$$
\dot{n}^{\mathrm{G}}=\left(1-n^{\mathrm{G}}\right)\left(\frac{\dot{p}^{\mathrm{sG}}}{K^{\mathrm{sG}}}+\operatorname{div}\left\langle\boldsymbol{v}_{\mathrm{G}}\right\rangle\right),
$$

with

$$
\dot{p}^{\mathrm{sG}}=-\frac{K_{\mathrm{dr}}^{\mathrm{G}}}{1-n^{\mathrm{G}}} \operatorname{div}\left\langle\boldsymbol{v}_{\mathrm{G}}\right\rangle+\frac{\zeta^{\mathrm{G}}-n^{\mathrm{G}}}{1-n^{\mathrm{G}}} \dot{p}^{\mathrm{fG}}
$$

and $\operatorname{div}\left\langle\boldsymbol{v}_{\mathrm{G}}\right\rangle=\operatorname{div}\langle\boldsymbol{v}\rangle+\dot{f}^{\mathrm{G}} / f^{\mathrm{G}}$.

The model is the backbone of our non-Lagrangian numerical method summarized in Sect. 4. Its is important to note that the set of equations (43) and (44) holds at each spatial point and at all interfaces, as well as for zones occupied by a single or multiple bulk materials. All materials that might be present in such processes, either compressible or incompressible, are treated in a unified fashion due to the decomposition of stress (5) used for all constituents. 


\subsection{Volume Fraction Evolution and Homogeneous Equilibrium}

In the mathematical sense of counting equations and unknowns, the above system of equations requires an additional $M-1$ closure relations (for the present case, $M=3$ ) to become a closed system. It is natural to think of this indeterminacy as being associated with conservation of mass and the evolution of the material volume fractions $f^{k}$, with $k \in\{1, \ldots, M\}=\{\mathrm{S}, \mathrm{F}, \mathrm{G}\}$. The reader is referred to $[44,45,51]$ for a more general discussion of volume fraction closure in two- and multi-phase flow models.

A simple way to obtain topological closure for multi-material flow is to assume constant volume fractions. However, such an assumption is inadequate if material compressibilities differ by several orders of magnitude. In our approach, we achieve closure by assuming homogeneous equilibrium [53,116, 149], formalized through

$$
p^{k}=\langle p\rangle \quad \text { and } \quad \boldsymbol{v}^{k}=\langle\boldsymbol{v}\rangle \quad \text { for all } k \in\{\mathrm{S}, \mathrm{F}, \mathrm{G}\} .
$$

The first assumption of pressure equilibrium between bulk materials is adequate because the speed of sound in each material is large compared with velocities in the problems of interest. In other words, equilibration is infinitely fast such that pressure is continuous across a material interface. The second assumption, $(52)_{2}$, has already motivated zero momentum exchange between the constituents; see (40). However, it is not generally a reasonable one because equilibration of velocity differences is driven by drag forces on material interfaces. Using the assumption implies fully bonded materials.

In following $[53,116]$ and using the assumptions $(52)$, have derived in $[17,21]$ a more realistic closure model reflecting homogeneous equilibrium:

$$
\dot{f}^{k}=f^{k}\left(\frac{\langle K\rangle}{K^{k}}-1\right) \operatorname{div}\langle\boldsymbol{v}\rangle, \quad \text { for all } k \in\{\mathrm{S}, \mathrm{F}, \mathrm{G}\} .
$$

Note that this equation naturally provides for a mixture compression model and a void collapse mechanism: the material with the smallest bulk modulus contributes most to the total volume change.

\subsection{Non-Lagrangian Formulation of the Model}

Since local volume averaging is defined with respect to a fixed region of space, the Eulerian formulation of the proposed model is natural. Indeed, the Lagrangian form (43) and (44) has been derived from the Eulerian conservation form (41) and (42), respectively, using the material time derivative. The arbitrary LagrangianEulerian (ALE) formulation further generalizes the model. A condensed derivation is given below; for more details, see $[13,14]$.

According to Sect. 1.3, the ALE formulation introduces a reference domain which may move in space at an arbitrary velocity $\boldsymbol{w}$. The relative volume change between the referential coordinate system and the spatial coordinate system is the Jacobian, $J$, and its rate of change is given by

$$
\frac{\partial J}{\partial t}=J \operatorname{div} \boldsymbol{w} .
$$


The ALE operator (1) is substituted into the equations (43) and (44), respectively. Multiplication of the resulting equations with $J$ and substitution of (54) gives the ALE conservation form of (43) and (44):

$$
\begin{gathered}
\frac{\partial \hat{p} J}{\partial t}+J \operatorname{div}(p \boldsymbol{c})=J(p-K) \operatorname{div} \boldsymbol{v} \\
\frac{\partial \hat{\rho} \hat{\boldsymbol{v}} J}{\partial t}+J \operatorname{div}(\rho \boldsymbol{v} \otimes \boldsymbol{c})=J(\rho \boldsymbol{b}+\operatorname{div}(\boldsymbol{s}-p \boldsymbol{I})),
\end{gathered}
$$

where

$$
c=v-w
$$

is the convective velocity. Angle brackets indicating mixture quantities have been dropped for notational brevity.

\subsection{Application: Isotropic Compression}

Consider a Lagrangian control volume occupied with a mixture of steel (bulk solid), air (bulk fluid), and dry sand. The volume is subjected to quasi-static isotropic compression. Under these conditions only (43) needs to be integrated in time. A first-order explicit scheme has been implemented for this purpose. The initial volume fractions are set to $f_{0}^{\mathrm{S}}=0.4, f_{0}^{\mathrm{F}}=0.2$, and $f_{0}^{\mathrm{G}}=0.4$, respectively, and the initial porosity of the sand is $n_{0}^{\mathrm{G}}=0.4$. The sand is chosen to be uniform and fine-grained, with an angle of internal friction $\phi=32^{\circ}$. The initial pressure of all constituents is the atmospheric pressure at sea level, $p_{\text {atm }}=101.0 \mathrm{kPa}$.

Steel under isotropic compression can be approximated by hypoelasticity [159]. The constant bulk modulus is $K^{\mathrm{S}}=1.6 \times 10^{8} \mathrm{kPa}$. Moreover, we assume that the compressibility of the bulk air and the air trapped in the sand pores does not change with pressure, so that $K^{\mathrm{F}}=K^{\mathrm{fG}}=p_{\text {atm }}=$ const.

Janbu's power law [173] is employed in order to model nonlinear stiffness of the granular material:

$$
E_{\mathrm{s}} \stackrel{\text { def }}{=} C\left(\frac{-\sigma^{\prime}}{p_{\text {atm }}}\right)^{a} p_{\text {atm }},
$$

where $E_{\mathrm{s}}$ is the confined stiffness of the bulk granular material measured in the confined compression (oedometer) test. $\sigma^{\prime}$ is the effective stress component in loading direction and $C, a$ are constants. For uniform fine sand, the values $C=300$ and $a=0.6$ are reasonable. Jaky's formula [55] then relates $\sigma^{\prime}$ in the oedometer test to the mean effective stress, yielding

$$
\sigma^{\prime}=-\frac{3\langle p\rangle}{1+2(1-\sin \phi)} .
$$

Finally, a relationship between $E_{\mathrm{s}}$ and the drained bulk modulus of the granular material, $K_{\mathrm{dr}}^{\mathrm{G}}$, can be established by using elasticity theory,

$$
K_{\mathrm{dr}}^{\mathrm{G}}=E_{\mathrm{s}} \frac{1+\nu}{3(1-\nu)},
$$




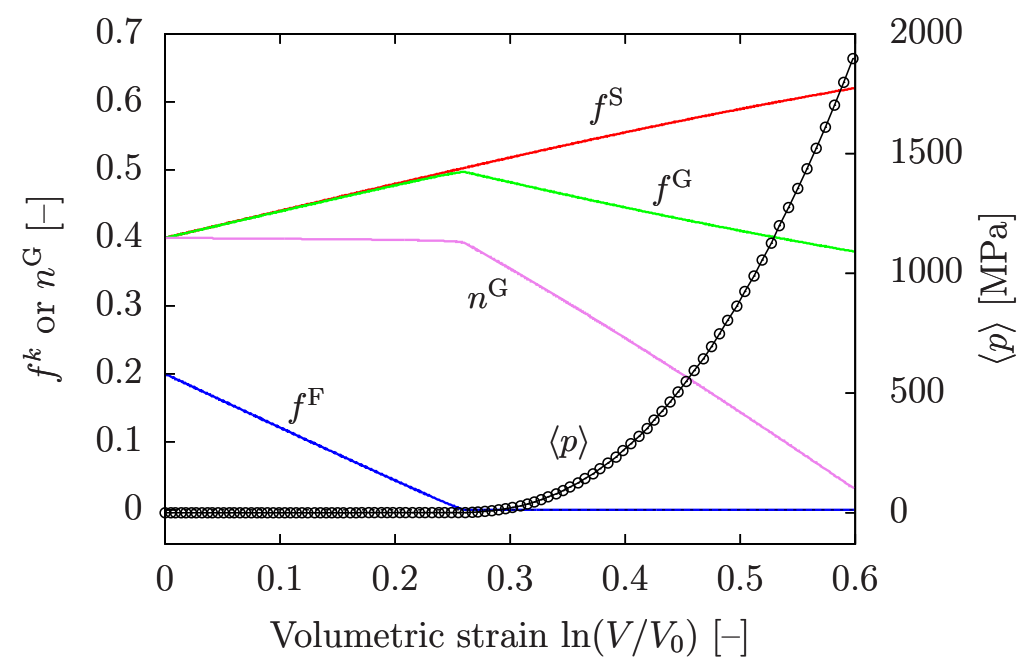

Fig. 4. Example application of the homogeneous equilibrium model: mixture of steel (bulk solid), air (bulk fluid), and dry sand under quasi-static isotropic compression. Evolution of the volume fractions, sand porosity, and mixture pressure. Reprint from $[17$, p. 97] with permission of Elsevier.

in which Poisson's ratio is estimated from $\nu=(1-\sin \phi) /(2-\sin \phi)$.

Results are plotted in Fig. 4 and seem to be reasonable. At the beginning of isotropic compression of the mixture, the bulk air is compressed, which does not significantly change the mixture pressure and the porosity of the granular material. The increase in bulk solid and granular material volume fractions is approximately the same due to (53) and the fact that the mixture bulk modulus is relatively low at this stage of compression. Once the bulk air has been completely compressed, mixture pressure increases exponentially because of the power law (58) and continuous compaction of the granular material. This, in turn, gives more weight to the large bulk modulus of steel in evaluating the mixture bulk modulus, (48).

\section{Numerical Techniques}

The MMALE method developed in the context of Subproject 5 is an extension of our simplified ALE method for plane and axisymmetric problems [14, 16, 19, 20, 140]. The implementation uses the three step scheme introduced in Sect. 1.4 and illustrated in Fig. 3. In this section we present the basic numerical techniques in the Lagrangian step, rezone step, and remap step associated with that scheme. Additional details can be found in $[18,141]$.

Let us write the set of equations (55) and (56) in compact form

$$
\frac{\partial \hat{q} J}{\partial t}+J \operatorname{div} F=S J
$$


where $q \in\{\rho \boldsymbol{v}, p\}, F$ is the convective flux of $q$, and $S$ is the source term. Conceptually, the three step scheme splits (61) into two sets of equations which are solved sequentially:

$$
\begin{aligned}
\frac{\partial \hat{q} J}{\partial t} & =S J, & & \text { (Lagrange) } \\
\frac{\partial \hat{q} J}{\partial t}+J \operatorname{div} F & =0 . & & \text { (remap) }
\end{aligned}
$$

The first set of equations, (62), is associated with $\boldsymbol{c}=\mathbf{0}$ resp. $\boldsymbol{v}=\boldsymbol{w}$. Hence, it is equivalent to the set of equations (43) and (44) and formalizes an Lagrangian description of motion. The solution of the second set of equations, (63), is associated with the remap step.

\subsection{Lagrangian Step}

During the Lagrangian step, the set (43) and (44) subject to prescribed initial conditions and boundary conditions is solved with almost standard finite element methods $[27,99,174,176]$. Accordingly, the set of equations is written in a weak form which is discretized in space using finite elements. A two-field mixed element formulation is used which accounts for material and geometric nonlinearities. For a single finite element $\Omega$, the weak form of the governing equations discretized in space can be written in matrix form

$$
\boldsymbol{M}^{*} \ddot{\boldsymbol{y}}+\boldsymbol{C}^{*} \dot{\boldsymbol{y}}+\boldsymbol{F}^{\text {in }}-\boldsymbol{F}^{\mathrm{ex}}=\mathbf{0} \quad \stackrel{\text { def }}{=} \boldsymbol{\Psi},
$$

in which

$$
\begin{aligned}
& \boldsymbol{\Psi} \stackrel{\text { def }}{=}\left[\begin{array}{l}
\Psi^{u} \\
\Psi^{p}
\end{array}\right], \quad \boldsymbol{y} \stackrel{\text { def }}{=}\left[\begin{array}{l}
u \\
p
\end{array}\right], \quad \boldsymbol{M}^{*} \stackrel{\text { def }}{=}\left[\begin{array}{rr}
\boldsymbol{M} & 0 \\
\mathbf{0} & 0
\end{array}\right], \\
& \boldsymbol{C}^{*} \stackrel{\text { def }}{=}\left[\begin{array}{cc}
\boldsymbol{C} & \mathbf{0} \\
\boldsymbol{Q}^{\mathrm{T}} & \boldsymbol{S}
\end{array}\right], \quad \boldsymbol{F}^{\text {in }} \stackrel{\text { def }}{=}\left[\begin{array}{c}
\boldsymbol{f}^{\text {in }}-\boldsymbol{Q p} \\
\mathbf{0}
\end{array}\right], \quad \boldsymbol{F}^{\mathrm{ex}} \stackrel{\text { def }}{=}\left[\begin{array}{c}
\boldsymbol{f}^{\mathrm{ex}} \\
\mathbf{0}
\end{array}\right], \\
& \boldsymbol{M} \stackrel{\text { def }}{=} \int_{\Omega} \boldsymbol{N}_{\boldsymbol{v}}^{\mathrm{T}} \rho \boldsymbol{N}_{\boldsymbol{v}} \mathrm{d} v, \quad \boldsymbol{Q} \stackrel{\text { def }}{=} \int_{\Omega} \boldsymbol{B}^{\mathrm{T}} \boldsymbol{m} \boldsymbol{N}_{p} \mathrm{~d} v,
\end{aligned}
$$

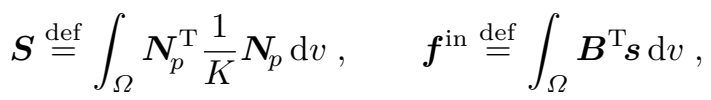

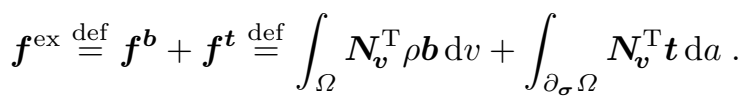

In the equations, $\boldsymbol{y}$ is the vector of nodal degrees of freedom, including the nodal pressure vector $\boldsymbol{p}$ and nodal displacement vector $\boldsymbol{u}, \boldsymbol{M}$ is called the (consistent) mass matrix, $\boldsymbol{Q}$ is the coupling matrix, $\boldsymbol{S}$ is the compressibility matrix, $\boldsymbol{f}^{\mathrm{in}}$ is the vector of internal nodal forces, and $f^{\text {ex }}$ is the vector of applied external nodal forces, consisting of the body forces $\boldsymbol{f}^{\boldsymbol{b}}$ and the surface forces $\boldsymbol{f}^{t}$. Moreover, $\boldsymbol{N}_{p}$ and $\boldsymbol{N}_{\boldsymbol{v}}$ are the matrices of the interpolation functions for pressure and velocity, respectively, $\boldsymbol{B}$ is the strain operator matrix, and $\boldsymbol{m}=[1,1,1,0]^{\mathrm{T}}$. A damping 
matrix $\boldsymbol{C}$ has been included for reasons of generality. If $\boldsymbol{C}=\mathbf{0}$, then damping is assumed to reside entirely in dissipative material behavior.

We emphasize that the terms in (64) are functions of the current geometry $\boldsymbol{x}$, which is also unknown. However, the current geometry can be determined from the initial geometry and the nodal displacements $\boldsymbol{u}$ due to the Lagrangian formulation. The material time derivative of the displacement is the velocity, that is, $\boldsymbol{v}=\dot{\boldsymbol{u}}$ and $\boldsymbol{v}=\ddot{\boldsymbol{u}}$ is the material acceleration.

The continuous time interval of interest, $[0, T] \subset \mathbb{R}$, is partitioned into a sequence of discrete time steps, using an incremental decomposition $t_{n+1}=$ $t_{n}+\Delta t$. Then, the solution of the semi-discrete weak form (64) is advanced implicitly in time using the Newmark-beta and generalized trapezoidal methods in conjunction with a damped Newton-Raphson method. The final form of the linearized system of equations reads

$$
\boldsymbol{K}_{n+1}^{i} d \boldsymbol{y}_{n+1}^{i}=\boldsymbol{r}_{n+1}^{i}
$$

where $i$ denotes the current iteration step, $\boldsymbol{r}_{n+1}^{i}$ is called the vector of residuals,

$$
\begin{aligned}
\boldsymbol{K}_{n+1}^{i} \stackrel{\text { def }}{=}\left[\begin{array}{cc}
\frac{\partial \boldsymbol{\Psi}^{\boldsymbol{u}}}{\partial \boldsymbol{u}_{n+1}^{i}} & \frac{\partial \boldsymbol{\Psi}^{\boldsymbol{u}}}{\partial \boldsymbol{p}_{n+1}^{i}} \\
-\frac{1}{a_{1}} \frac{\partial \boldsymbol{\Psi}^{\boldsymbol{p}}}{\partial \boldsymbol{u}_{n+1}^{i}} & -\frac{1}{a_{1}} \frac{\partial \boldsymbol{\Psi}^{\boldsymbol{p}}}{\partial \boldsymbol{p}_{n+1}^{i}}
\end{array}\right] \\
=\left[\begin{array}{cc}
a_{0} \boldsymbol{M}_{n+1}^{i}+a_{1} \boldsymbol{C}_{n+1}^{i}+\boldsymbol{D}_{n+1}^{i} & -\boldsymbol{Q}_{n+1}^{i} \\
-\left(\boldsymbol{Q}_{n+1}^{i}\right)^{\mathrm{T}} & -\frac{a_{6}}{a_{1}} \boldsymbol{S}_{n+1}^{i}
\end{array}\right]
\end{aligned}
$$

is the effective stiffness matrix,

$$
\boldsymbol{D} \stackrel{\text { def }}{=} \int_{\Omega} \boldsymbol{B}^{\mathrm{T}} \boldsymbol{c} \boldsymbol{B}_{\mathrm{dev}} \mathrm{d} v
$$

is the material stiffness matrix (ignoring initial stress stiffness), $\boldsymbol{B}_{\mathrm{dev}}$ is the deviatoric strain operator, and $a_{0}, a_{1}, a_{6}$ are numbers associated with the time integration scheme.

The element type used in the current implementation is the MINI quadrilateral element [24], which is the quadrilateral analogue of the MINI triangle element $[11,177]$. It is a stabilized quadrilateral using a bilinear approximation of the geometry, displacement, and pressure together with an additional bubble function for the displacement approximation.

Solution of the finite element system of equations (66) requires evaluation of the stress at the quadrature points of the elements in every iteration of every time step. The stress has to be integrated because the constitutive equations are generally given in spatial rate form using objective stress rates; cf. (15). Our current method employs an incrementally objective integration algorithm originally developed by Hughes [85] and improved in [147, sect. 8.3] in order to ensure exact stress update if the motion over a time step is rigid. 


\subsection{Rezone Step}

After the Lagrangian step, the mesh is rezoned. The rezone step provides the mesh velocity $\boldsymbol{w}$, from which the convective velocity required for remap can be determined in accordance with (57); cf. [14,15] for mesh rezoning resp. smoothing algorithms. If the MMALE method is run in the pure Eulerian mode $(\boldsymbol{w}=\mathbf{0}$ resp. $\boldsymbol{c}=\boldsymbol{v}$ ), the mesh nodes are relocated to their original positions. In either case the mesh topology remains unchanged.

\subsection{Remap Step}

The basic equation for the remap is the conservation law (63). Data assumed to be given in the remap step includes both the deformed geometry $\boldsymbol{x}^{-}$and rezoned geometry $\boldsymbol{x}^{+}$as well as the solution variables $q^{-}$obtained after the Lagrangian step; quantities before and after the remap are marked with - and + , respectively.

Due to the structure of (63), the remap takes the form of an advection problem subject to the initial condition $\left.q\right|_{t=t^{-}}=q^{-}$. A finite volume method $[14,20,98]$ is employed for numerical solution. Finite volume methods are conservative because they solve the integral form of a conservation law with respect to a control volume tessellation. In our method the control volume tessellation coincides with the underlying finite element mesh, hence the terms "control volume" and "element" can be used interchangeably. The problem for which we seek an approximate solution can be summarized as

$$
\frac{\mathrm{d}}{\mathrm{d} t} \int_{\Omega} q \mathrm{~d} v+\sum_{I} \int_{\Gamma_{I}} q \boldsymbol{c} \cdot \boldsymbol{n} \mathrm{d} a=0
$$

subject to $\left.q\right|_{t=t^{-}}=q^{-}$and for each element, where $\Omega$ is the element domain, $\Gamma_{I}$ is the boundary edge starting at local node $I$, with $\partial \Omega \approx \bigcup_{I} \Gamma_{I}$, and $\boldsymbol{n}$ is the unit normal to that edge.

After discretization in space and first-order explicit discretization in time, (69) takes the form

$$
q^{+}=\frac{q^{-} V^{-}-\sum_{I} \Delta Q_{I}^{-}}{V^{+}}, \quad \text { with } \quad V^{+}=V^{-}-\sum_{I} \Delta V_{I}
$$

Here $q^{+}$is the remapped field under consideration, $V^{-}$is the volume of the deformed element in the Lagrangian mesh, $V^{+}$is the element volume in the relocated mesh (i.e. after the remap), $\Delta V_{I}$ is the total volume transported across the edge $\Gamma_{I}$ between $\Omega$ and the element $\Omega_{\mathrm{adj}(I)}$ adjacent to $\Gamma_{I}$, and $\Delta Q_{I}^{-}$represents the transported $q$-volume across that edge.

For each element, $\Delta V_{I}$ is commonly defined as the volume swept out by the edge $\Gamma_{I}$ between the configurations $\boldsymbol{x}^{-}$and $\boldsymbol{x}^{+}$[39]. Moreover, it is defined positive if the nodes defining the edge are moved further into the element's 
region, that is, if the transport volume is leaving the element. The transported $q$ volume $\Delta Q_{I}^{-}$, on the other hand, is approximated by using the so-called Godunov flux

$$
\begin{aligned}
\Delta Q_{I}^{-} & \stackrel{\text { def }}{=} \frac{1}{2} \Delta V_{I}\left(q^{-}+q_{\mathrm{adj}(I)}^{-}\right)+\frac{1}{2}\left|\Delta V_{I}\right|\left(q^{-}-q_{\mathrm{adj}(I)}^{-}\right) \\
& =\frac{1}{2}\left(\left(q^{-}+q_{\mathrm{adj}(I)}^{-}\right)+\operatorname{sgn}\left(\Delta V_{I}\right)\left(q^{-}-q_{\mathrm{adj}(I)}^{-}\right)\right) \Delta V_{I} .
\end{aligned}
$$

Substitution into (70) results in a conservative first-order upwind transport algorithm which corresponds to the classical donor-cell difference scheme [34]. Donor-cell advection is used in many ALE codes because it is simple, stable, conservative, and monotonicity-preserving [33,61,69,70,82,129]. Discussions of this and several other advection algorithms can be found, for example, in [34,80,98].

The pseudocode of a suitable implementation of the donor-cell transport algorithm in the remap step is provided in Alg. 1, and it uses a procedure outlined in [39]. Note that volume is updated only if the total transported volume is positive, i.e. a negative transport volume is set to zero, and a volume subtracted from the element is added to element $\operatorname{adj}(I)$ adjacent to edge $I$ to avoid double counts. This eliminates half of the remap operations. Higher-order accurate transport algorithms have the same structure as the donor-cell algorithm Alg. 1, except for the evaluation of $q^{-}$occurring in line 10 [39].

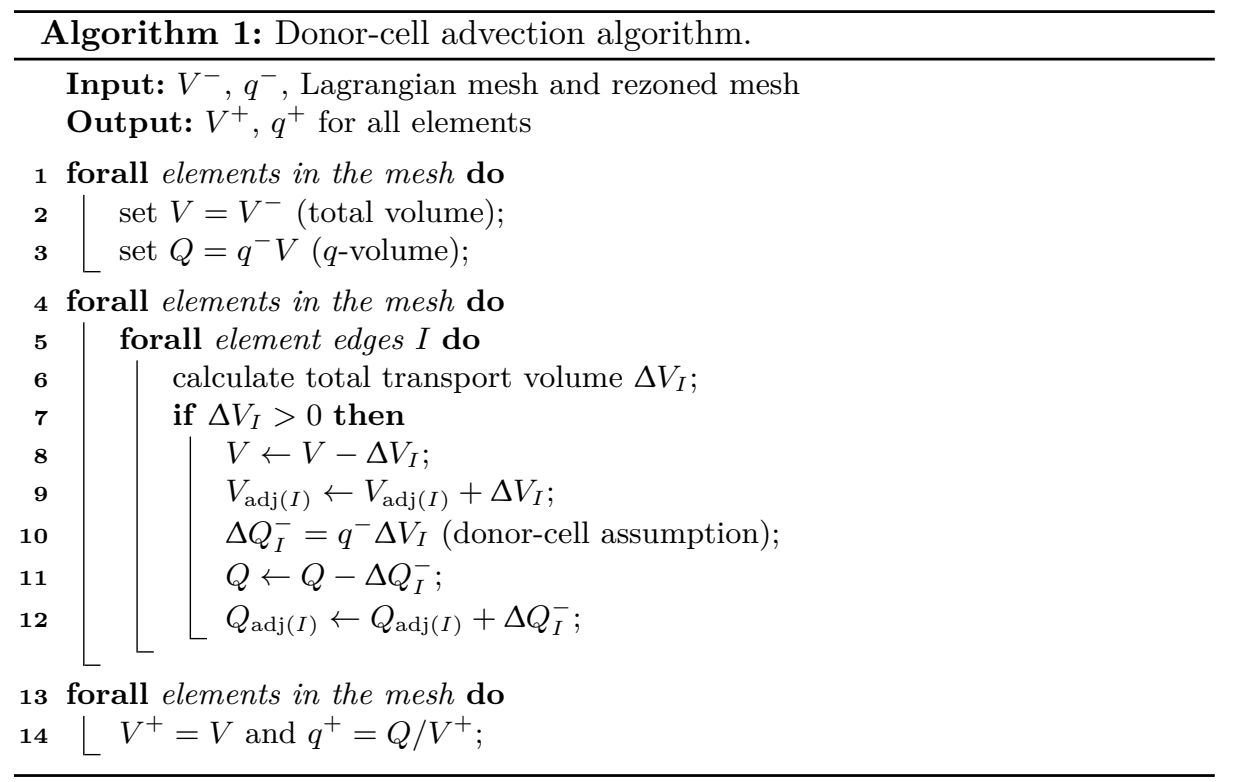




\subsection{Treatment of Multi-Material Elements}

One important feature that distinguishes non-Lagrangian from the Lagrangian numerical methods is the presence of multi-material elements (cf. Sect. 1 and Fig. 3 right). These elements require extensions of the Lagrangian step and remap step presented in the previous sections in order to achieve a reasonable accuracy of the overall MMALE method.

Mixture Model in the Lagrangian Step In the Lagrangian step, the stress and state variables are integrated in time for each individual material. Thereafter, the stress and stiffness for the mixture of materials has to be evaluated and substituted into the respective terms of the system of finite element equations (66). For this purpose, we have developed the framework and homogeneous equilibrium model summarized in Sect. 3 accounting for the two-phase behavior of saturated granular material. We reemphasize that the homogeneous equilibrium model is consistent with the set of equations solved in the Lagrangian step, hence valid for both single- and multi-material elements.

Eulerian and MMALE meshes may consist of elements that partially or completely cover void (empty space or atmosphere); note that void is treated as a particular bulk fluid in (43) and (44). These elements require special treatment in the implicit Lagrangian step because a stiffness matrix has to be inverted [38]. Void elements practically do not have any stiffness or mass density. Therefore, their nodes remain unconsidered when setting up the finite element system of equations. Elements located at material boundaries are partially filled with void. The stiffness of the mixture inside these elements might be low, causing large displacement increments during the equilibrium iterations. Therefore, the incremental nodal displacements of partially filled elements are uniformly scaled.

Material Subzones Representation in the Remap Step If an element contains $m>1$ materials, the remap must be carried out for all variables $q$ of each individual material $k$, with $k \in\{1, \ldots, m\}$. Application of mixture theory (Sect. 3) then requires that the advected variable is $q^{k} f^{k}$, where $q^{k}$ represents the averaged variable measured per unit volume of the $k$-material, and $f^{k}$ is the $k$-material volume fraction. The generic advection algorithm (70) then takes the form

$$
q^{k+}=\frac{q^{k-} f^{k-} V^{-}-\sum_{I} \Delta Q_{I}^{k-}}{f^{k+} V^{+}} \text {for all } k \in\{1, \ldots, m\},
$$

and with $V^{+}$obtained from $(70)_{2}$.

For the determination of the transported (advected) volume fraction $f^{k+}$ and the transported $q^{k}$-volume $\Delta Q_{I}^{k-}$ across element edges $I$, the the spatial distribution of the material subzones (subcells) in multi-material elements must be taken into account. Otherwise initially coherent zones would disperse after a few advection steps. The present MMALE method reconstructs and propagates material interfaces element by element by using a volume of fluid (VOF) method. 
The amount of transported material is defined as the regions swept out by the element edges during mesh rezoning truncated by the interfaces.

Common state-of-the-art VOF methods approximate the interface in each multi-material element by a straight line; see reviews in $[37,137]$. One of the earliest two-dimensional methods is due to Youngs [175], which forms a basis for the developments of the present research. Our implementation relies on that described in [138] because the original paper provides little detail of the interface reconstruction procedure. Details are presented in [22], so only a few key facts will be repeated here.

A linear interface can be generally described by the Hesse normal form

$$
\boldsymbol{n} \cdot \boldsymbol{x}-d=0
$$

in which $\boldsymbol{x}$ is an arbitrary point on the interface, $\boldsymbol{n}$ is the unit normal on that interface, and $d$ is the line constant. A linear reconstruction of the interface is determined for each element in two steps: (i) estimate $\boldsymbol{n}$, or equivalently, the interface slope and (ii) determine $d$ such that the volume fraction of the material lying behind the interface matches the known value.

The interface slope is estimated based on the volume fraction data in the current element and its neighbors. The location of the material interface is determined by noting that the truncated element volume behind the interface represents the partial material volume. Volume is conserved, i.e. the right location of the interface has been determined, if the partial volume divided by the element volume matches the given volume fraction data of that element. The matching can be achieved by deriving an explicit expression that relates the truncated element volume to $d$.

The material transport volumes are usually computed as truncation volumes, as illustrated in Fig. 5. Once the total transport volume across an element edge, $\Delta V$, has been determined, a region having the same amount of volume is created that lies entirely within the element (Fig. 5 right). The $k$-material transport volume, $\Delta V^{k}$, is then defined as the set-theoretic intersection of the total transport volume and the material domain behind the reconstructed interface. Finally, the material volume fraction can be updated in accordance with (70), i.e.

$$
f^{k+}=\frac{f^{k-} V^{-}-\sum_{I} \Delta V_{I}^{k}}{V^{+}} .
$$

Once the transported $k$-material volumes $\Delta V_{I}^{k}$ are known, the transported $q^{k}$ volumes $\Delta Q_{I}^{k-}$ needed for the remap (72) can be determined by an appropriate advection scheme. In case of donor-cell advection (71),

$$
\Delta Q_{I}^{k-}=\frac{1}{2}\left(\left(q^{k-}+q_{\mathrm{adj}(I)}^{k-}\right)+\operatorname{sgn}\left(\Delta V_{I}^{k}\right)\left(q^{k-}-q_{\mathrm{adj}(I)}^{k-}\right)\right) \Delta V_{I}^{k} .
$$

\subsection{Rigid Disk in Uniform Flow Field}

To test the VOF reconstruction and propagation algorithms of our method, we consider a rigid circular disk transported in a uniform diagonal velocity field. 


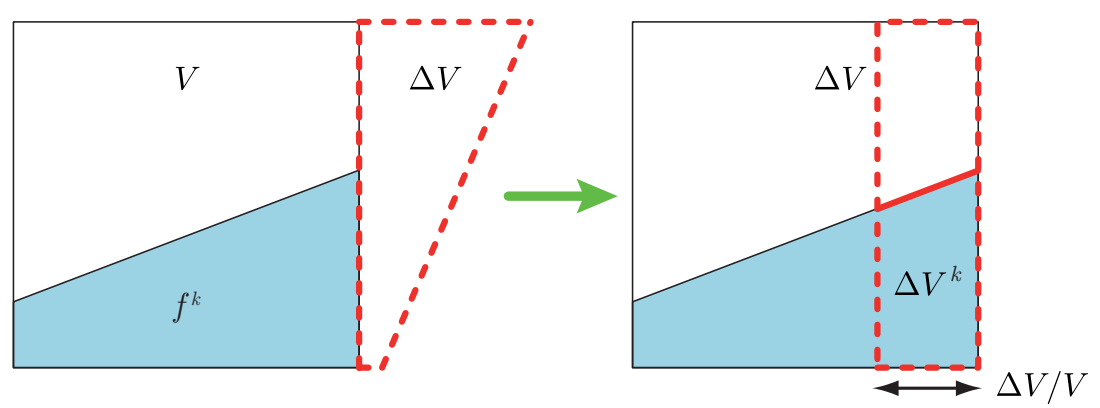

Fig. 5. VOF interface propagation in accordance with $[34,175]$. The reconstructed interface and the total volume $\Delta V$ transported across an element edge are used to calculate the transported material volume $\Delta V^{k}>0$.

The problem statement and square mesh are shown in Fig. 6a. Fig. 6b depicts the reconstructed interface in the initial configuration and the corresponding distribution of the red material volume fraction. The latter has been obtained by intersecting the circular material domain with the domain enclosed by each mesh element, resulting in a volume fraction constant in each element. A direct comparison of the original interface and its piecewise linear reconstruction is given in the detailed view of Fig. 7. Note that the fractional element volume on each side of the interface is the same for the original interface and its linear approximation.

The second and third row in Fig. 6 show the material configurations and volume fraction distributions after advecting the material along the diagonal of the computational domain. Figs. 6c and 6d plot the results for the case where the transport algorithm does not account for the geometry of the material subzone in each element. It can be seen that the material loses coherence and disperses during transport (Fig. 6c), leading to a blurred red zone in the volume fraction plot (Fig. 6d). In contrast to that, the shape of the circular disk is retained throughout the calculation if the material distribution is considered in each remap step by using the VOF algorithm (Figs. 6e and 6f).

\section{Experimental Tests}

Experimental model tests have been carried out in the context of Subproject 5 to observe the multi-material flow field during vibro-injection pile (RI-pile) installation in sand. This section summarizes the test set-up, measurement concept and experimental program, and discusses some preliminary results. Further details have been presented in a previous paper [141]. Moreover, we refer the reader to the benchmark tests done in the Central Project of the DFG Research Unit, e.g. those reported in $[162,163]$ and in this collection. 


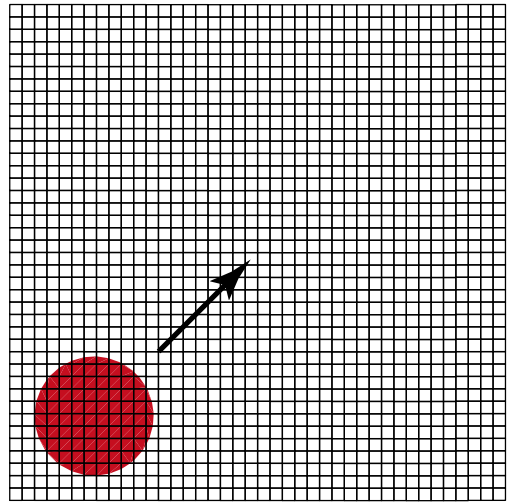

(a)

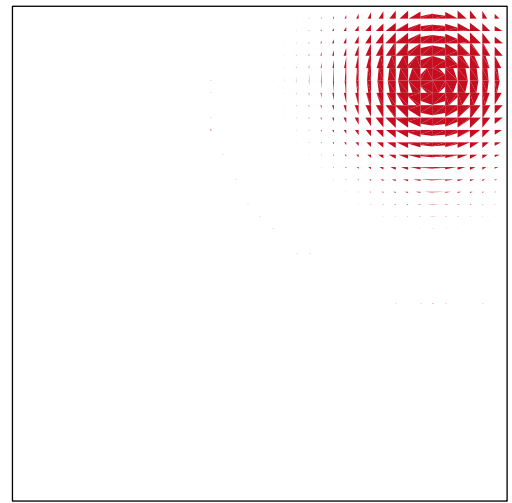

(c)

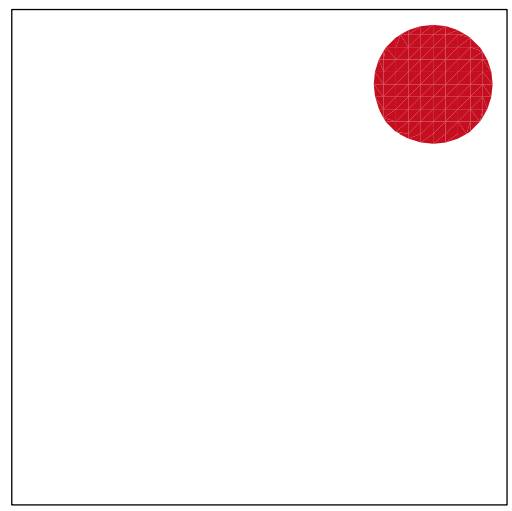

(e)

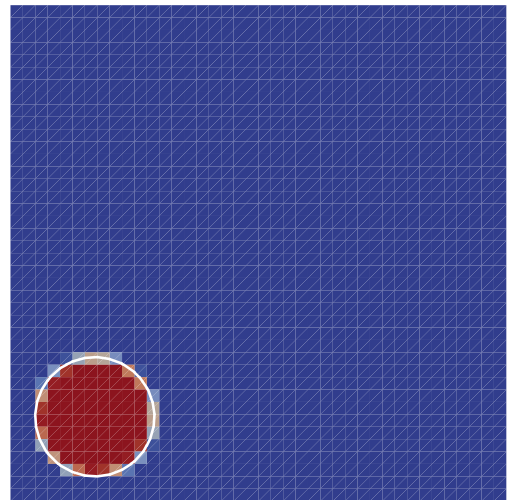

(b)

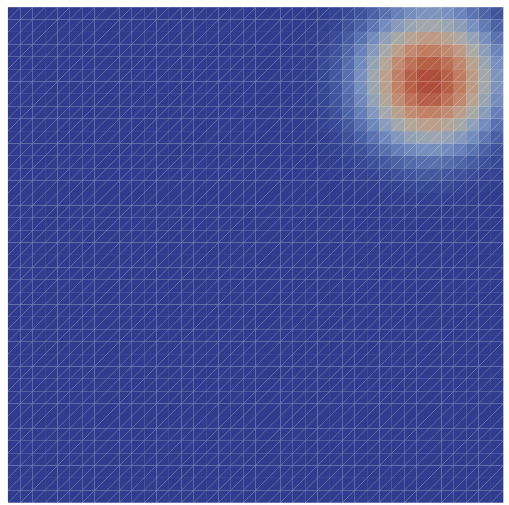

(d)

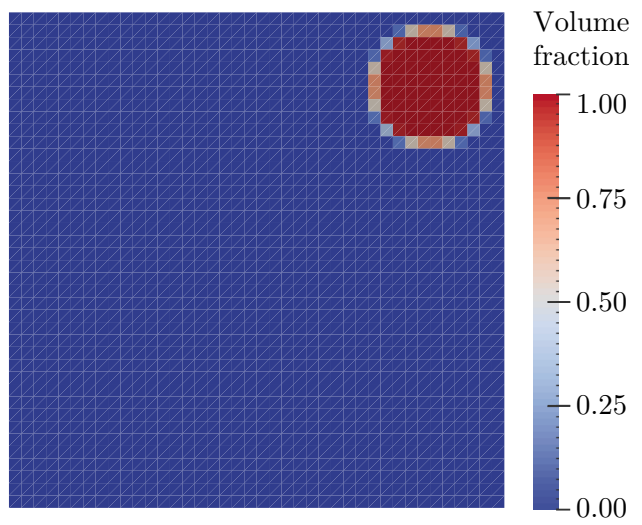

(f)

Fig. 6. Transport of a circular disk in uniform flow. (a) Initial configuration and mesh. (b) Initial volume fraction distribution and reconstructed material interface. (c) Final configuration and (d) final volume fraction distribution without interface tracking. (e) Final configuration and (f) final volume fraction distribution using the VOF method. 


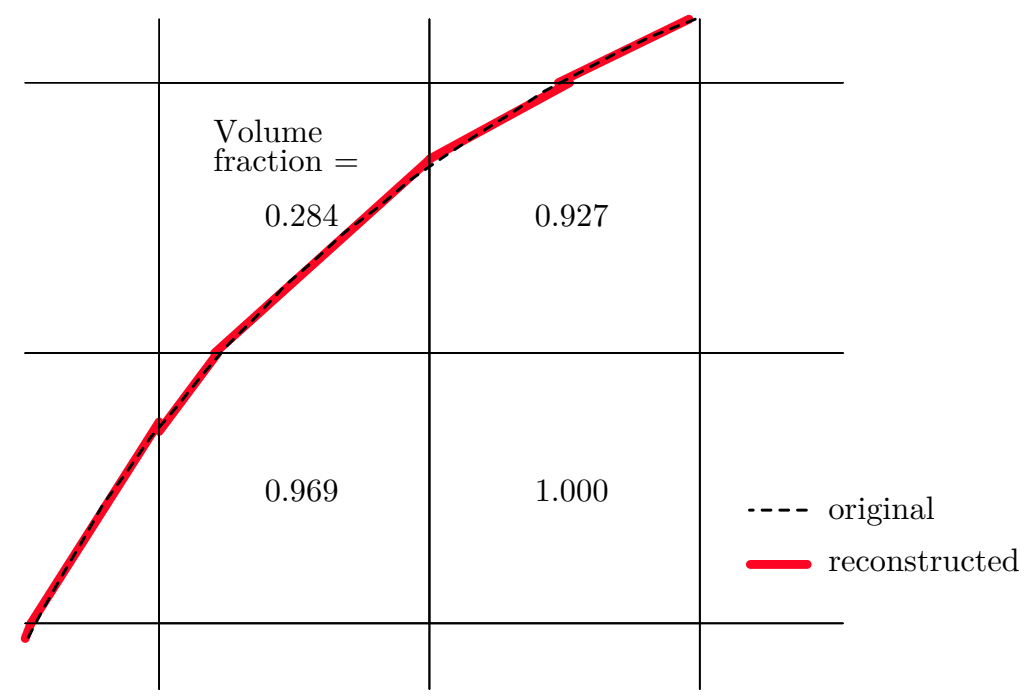

Fig. 7. Detailed view of Fig. 6b. Original interface portion (dashed line) and piecewise linear reconstructed interface (solid red line) which is generally discontinuous across element edges. The material volume fraction in each element matches the original value obtained by intersecting the circle with the element domain.

\subsection{Set-up and Measurement Concept}

The set-up used for the tests is shown in Fig. 8. The main components are a waterproof chamber with glass panel serving as a viewing window, a model pile, and a device for vibratory pile driving. All components are in-house developments. The vibrator consists of two counter-rotating imbalances whose mass and rotational speed are adjustable. The model pile is made up of a $50 \mathrm{~mm} \times 50 \mathrm{~mm}$ stainless steel square tube equipped with a welded-on collar at the pile toe and a built-in injection tube. The opening of the injection tube is located directly above the collar (Fig. 8c). Pressurized injection is enabled by a diaphragm pressure vessel.

During the tests the pile was guided alongside the glass panel. The driving and grouting process was digitally filmed through the viewing window using a standard Full HD camcorder. Series of consecutive still images were recorded at $50 \mathrm{~Hz}$ with a maximum resolution of $1920 \times 1080$ pixels. Digital still images of the configurations at the end of the tests were also captured. By analyzing the recorded image sequence using image correlation software, details of the multimaterial flow field could be measured without on-sample instrumentation. A MATLAB toolbox based on particle image velocimetry (PIV) $[4,14,171]$, called GeoPIV [170], is used in the present research. PIV tracks the texture within areas of an image through a sequence of images to determine local incremental displacement vectors. The totality of these displacement vectors represents an 

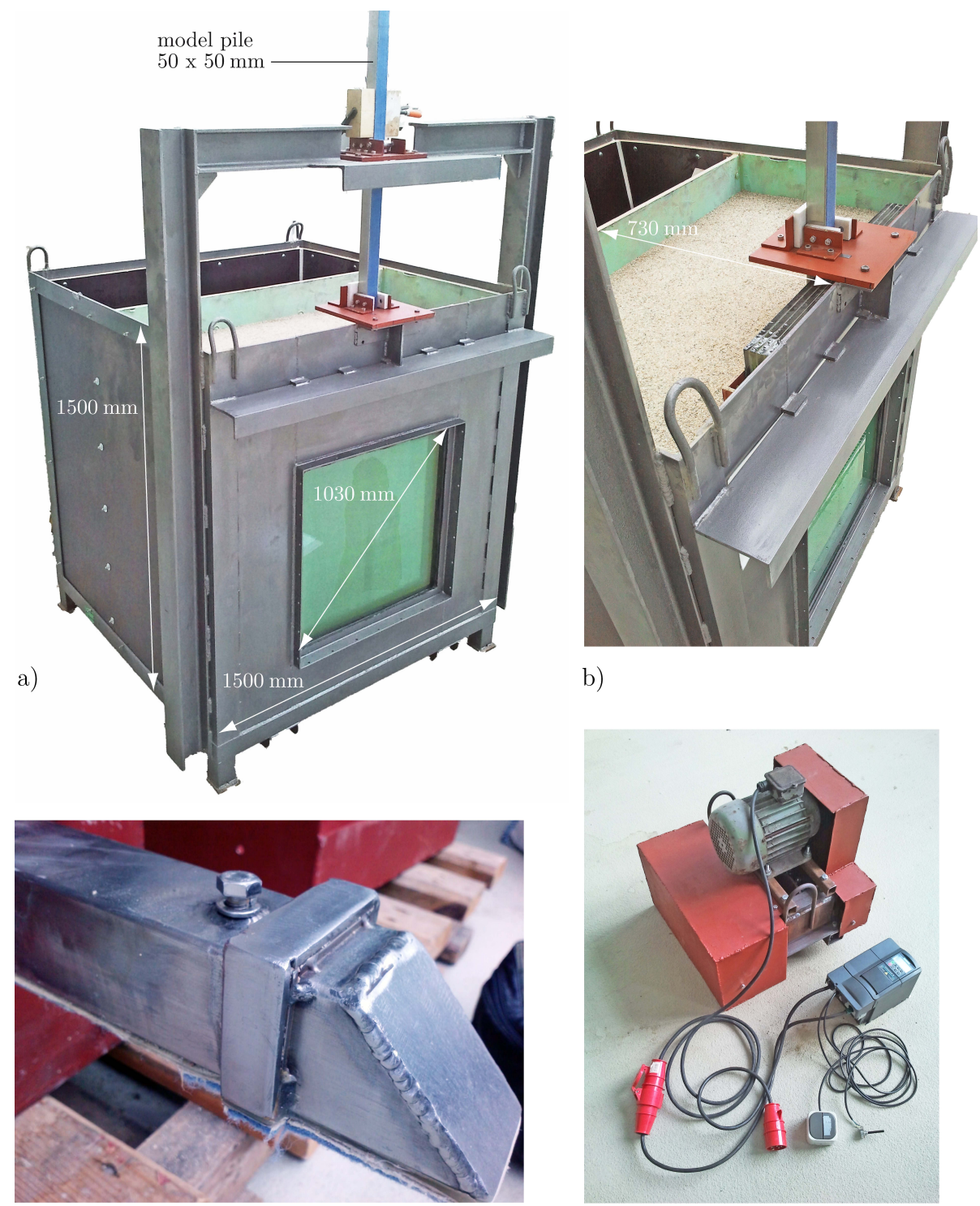

b)

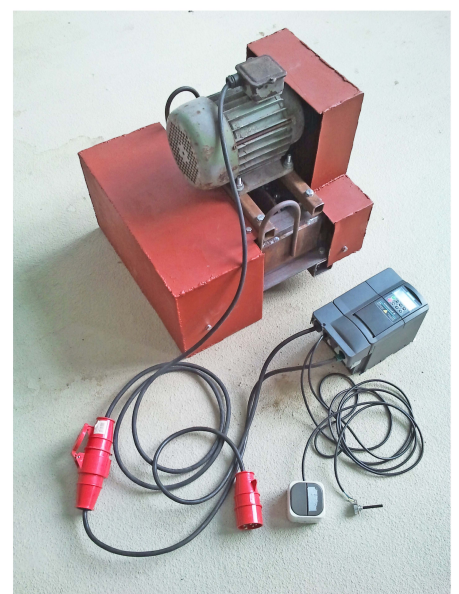

c)

d)

Fig. 8. Experimental investigation of vibro-injection pile installation. a) Filled test chamber with glass panel and model pile. b) Detailed view of the glass panel, pile guide, and model pile. c) Tapered pile toe with welded-on collar and bolt closing the injection tube. d) Self-made vibrator with controller (frequency converter). Reprint from [141, p. 117] with permission of Springer. 
incremental displacement field from which an incremental strain field can be obtained through postprocessing.

\subsection{Experimental Program}

The sand employed in the experimental model tests is a quartz sand with wellrounded to angular grains identified as fine-gravelly coarse Sand (fgrCSa) according to [62]. The limit void ratios are $e_{\min }=0.482$ and $e_{\max }=0.779$. Further granulometric properties are listed in [14], in which the same sand was used for quasi-static penetration tests. In all tests the chamber was filled with air dried sand by dry sieve pluviation. Each sand model was prepared in several layers of equal thickness in order to achieve a homogeneous distribution of initial density. Due to geometric constraints of the chamber the initial mean relative density was always larger than $85 \%$ (very dense).

Table 1. Details of conducted vibro-injection pile (RI-pile) installation tests. Reprint from [141, p. 119] with permission of Springer.

\begin{tabular}{|c|c|c|c|c|}
\hline Test ID & Date & Saturation & $\begin{array}{l}\text { Grouting material / } \\
\text { Pressurization [bar] }\end{array}$ & $\begin{array}{c}\text { Frequency }[\mathrm{Hz}] / \\
\text { Load amplitude }[\mathrm{kN}]^{a}\end{array}$ \\
\hline RI-1-D & \multirow{4}{*}{2011} & air dried & \multirow{3}{*}{ none } & $20.7 / 2.56$ \\
\hline RI-2-F & & \multirow{3}{*}{ water flooded } & & $20.0 / 2.39$ \\
\hline RI-3-F & & & & $20.0 / 1.90$ \\
\hline RI-5-B ${ }^{b}$ & & & bentonite slurry $/-^{c}$ & $20.0 / 2.39$ \\
\hline "RI-6-D & \multirow[b]{2}{*}{2012} & air dried & none & \multirow[b]{2}{*}{$20.0 / 2.39$} \\
\hline $\begin{array}{c}\text { RI-7-B } \\
\text { RI-8-B } \\
\text { RI-9-H }\end{array}$ & & water flooded & $\begin{array}{l}\text { bentonite slurry / }-^{c} \\
\text { bentonite slurry / } 1.0 \\
\text { hydraulic binder / 3.0 }\end{array}$ & \\
\hline RI-10-H & 2013 & water flooded & hydraulic binder / 3.0 & $20.0 / 2.39$ \\
\hline
\end{tabular}

$\mathrm{D}$ - dry; $\mathrm{F}$ - flooded; $\mathrm{B}$ - bentonite; $\mathrm{H}$ - hydraulic binder

In all tests the initial mean relative density was $>85 \%$ (very dense).

${ }^{a}$ with respect to the vibrator; the static force (dead weight) varies between the tests

${ }^{b}$ analyzed by using particle image velocimetry

${ }^{c}$ loaded under its own weight

Series with a total of 10 tests have been conducted (Tab. 1). The degree of saturation, the grouting material, the grouting pressure, and the load amplitude of the vibrator were varied between the tests. The vibration frequency was about $20 \mathrm{~Hz}$ for all tests and the load amplitude varied between $1.9 \mathrm{kN}$ and $2.56 \mathrm{kN}$. Two tests were run in air dried sand, whereas the other were carried out in sand which had been water flooded. In three experiments the pile shaft annulus created by the welded-on collar was injected with pigmented bentonite slurry, whereas hydraulic binder was used in two other tests. 


\section{$5.3 \quad$ Test Results}

All the tests using bentonite slurry injection failed in keeping open the shaft annulus created by the collar at the model pile toe. In contrast to that, the hydraulic binder has a sufficiently high shear resistance to stabilize the shaft annulus while possessing excellent flowability during pressurized grouting. Fig. 9 shows two digital photographs of test RI-9-H recorded about half an hour after the pile installation has completed. Although the amount of hydraulic binder infiltration into the pores of the coarse test sand increases with time, it cannot be completely avoided. Therefore, the assumption of impermeable interfaces (zero mass exchange) in the mixture model summarized in Sect. 3 is not always a reasonable one. Despite this, Fig. 9 indicates a clear soil-grout interface which is almost vertical along the pile shaft.

Fig. 10 shows the results of a PIV analysis of test RI-9-H using hydraulic binder injection. Fig. 10a plots the time history of the vertical displacement of the pile tip. Those configurations where image capturing took place are marked with black squares. During a vibration cycle, however, the pile moves upward and downward. The displacement increments in the soil which occurred during the downward motion of the pile between image 1 and image 2 are displayed in Fig. 10b using vectors with scaled length. It is clearly visible that the soil is not only displaced below the pile toe and underneath the collar in a predominantly vertical direction but also moves downward above the collar. Figure 10c shows the displacement increments due to upward motion of the pile between image 5 and image 6 . The vectors above the collar indicate that the soil located at the soil-grout interface is dragged along with the pile motion and displaced in lateral direction. The heavings beneath the pile toe and the collar result from the release of the previously compressed soil.

\section{Conclusions and Outlook}

The original objective of Subproject 5 of the DFG Research Unit FOR 1136 GeoTech has been the numerical simulation of vibro-injection pile installation in sand. Our research work within this project, however, has revealed that available methods will not produce reliable results because of two shortcomings: (i) they cannot represent the large deformations and material interface evolution associated with such multi-material flow situations and/or (ii) they cannot accurately reproduce the complex two-phase, coupled behavior of saturated geomaterial. Consequently, we adjusted the focus of our research towards theoretical modeling of geotechnical and geomechanical processes in general, and the development of a numerical tool that consistently employs the advanced models in a non-Lagrangian formulation.

A rigorous theoretical framework has been developed in order to model the geomaterial (saturated sand or debris material) as a general grain-fluid mixture as well as its interaction with multiple other bulk materials such as pure solid and 

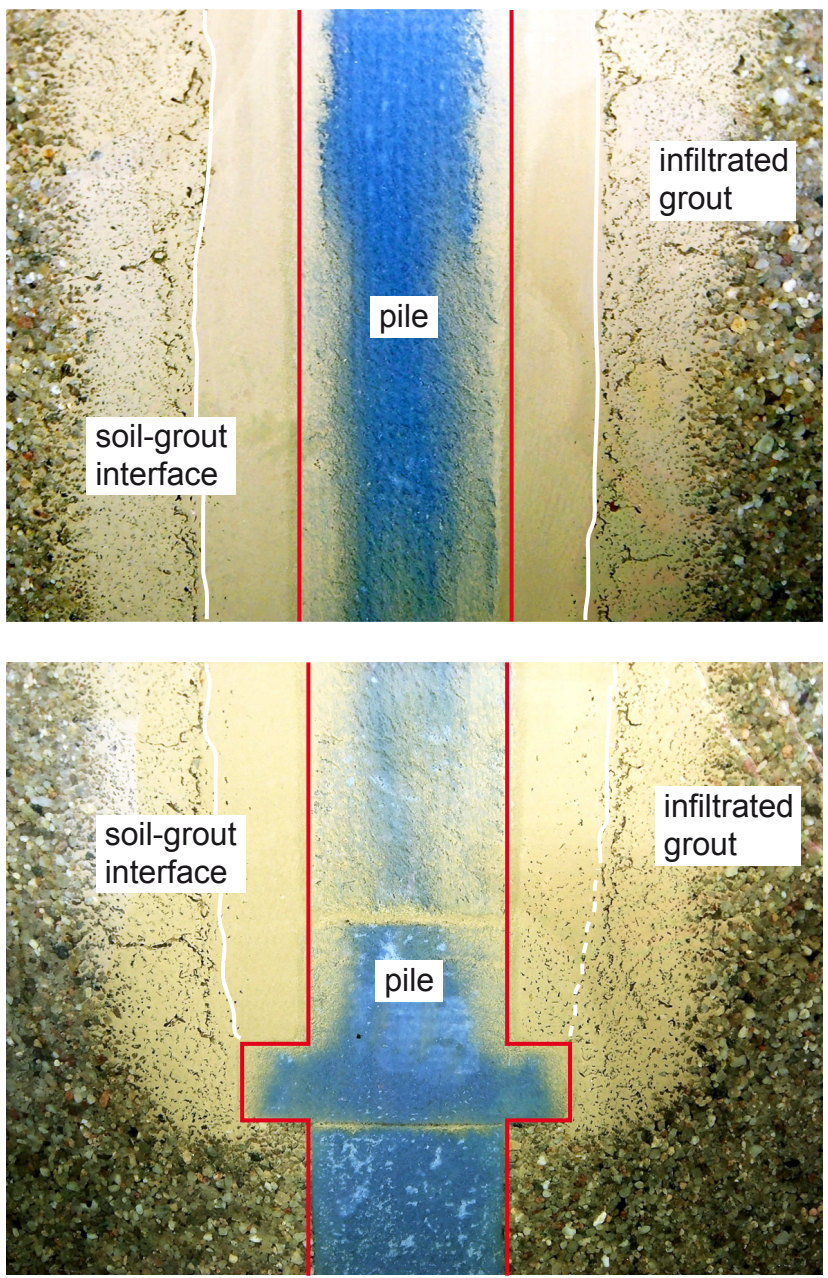

Fig. 9. Digital photograph of the configuration of test RI-9-H (hydraulic binder injection) through the viewing window of the chamber about half an hour after pile installation has completed. Reprint from [141, p. 120] with permission of Springer. 


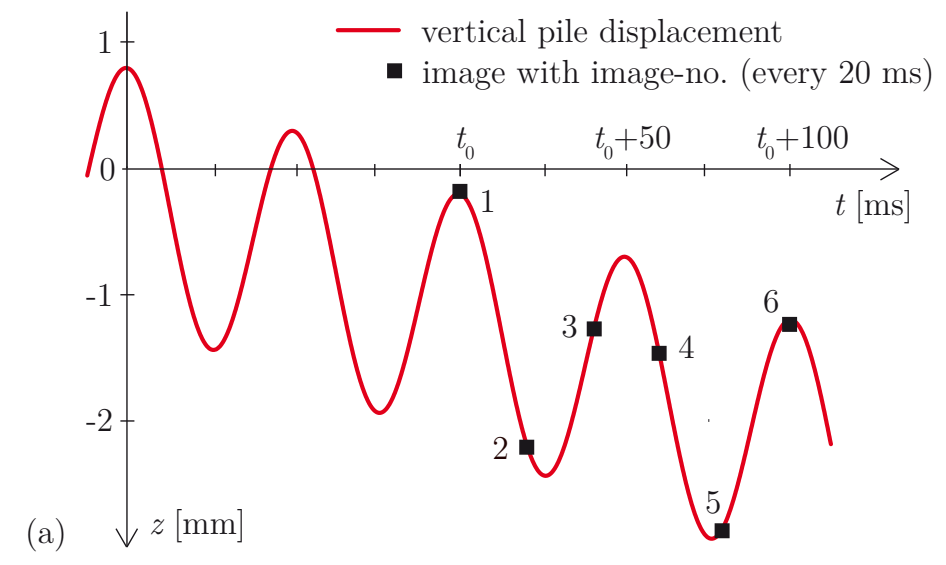

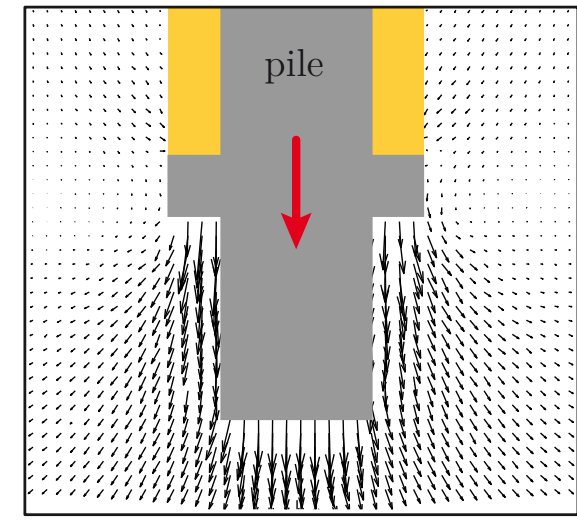

(b)

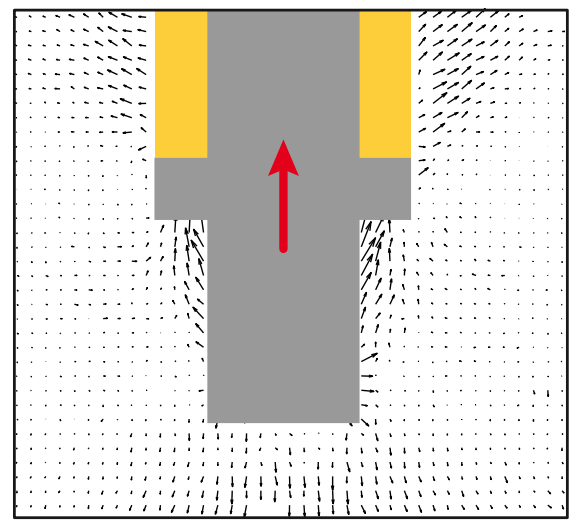

(c)

Fig. 10. Results of model test RI-9-H using hydraulic binder injection. (a) Schematic time history of vertical pile displacements. PIV results showing soil displacement increments (b) at downward motion of the pile (image 1 to image 2), and (c) at upward motion of the pile (image 5 to image 6 ). 
pure fluid on the macroscale. Macroscopic balance principles have been derived from the corresponding balance principles on the microscale by employing local volume averaging as the filtering technique. In contrast to common two-scale theories, the proposed three-scale hybrid mixture approach is able to incorporate both the evolution of bulk material interfaces as well as the two-phase phenomena associated with grain-fluid mixtures. Moreover, the approach allows for the use of phenomenological constitutive models describing grain-fluid mixture response for different flow regimes. Accordingly, the stress tensors have been split into rate-independent and rate-dependent parts, and into a pressure stress and an extra stress. Closure of the mixture model has been achieved by the fundamental assumption of homogenous distributions of pressure and velocity.

To numerically model and simulate geotechnical and geomechanical processes, we have developed a multi-material ALE (MMALE) method. The implementation of the method is based on the common three step scheme, splitting incremental solution into Lagrangian, rezone, and remap steps. MMALE allows material interfaces to flow through the computational mesh, so that multimaterial elements may arise which contain two or more materials. A homogeneous equilibrium mixture model derived from the proposed hybrid mixture theory describes the interaction within those multi-material elements in a mechanically consistent way. The donor-cell advection algorithm is used to conservatively remap the solution variables onto the rezoned mesh. To precisely determine the amount of lost or gained material volume, material interfaces are reconstructed and propagated through the mesh by using the VOF technique.

Experimental model tests have been carried out in order to investigate the relevant phenomena of vibro-injection pile installation in sand, an example of a complex geotechnical process. A special model pile and a test chamber with viewing window have been designed and manufactured for this purpose. Image sequences have been recorded through the viewing window during the installation process, which have been subsequently analyzed by using particle image velocimetry. The tests indicate that displacement, infiltration, and mixing occurs along the soil-grout interface due to the dynamical interaction of multiple, physically distinct materials on different spatial scales. The tests also reveal that the grains in a body of sand usually entail adequate image texture for PIV analysis, but not the grouting material unless it would be seeded with marker particles.

Future work will focus on the application of the the MMALE method in conjunction with the proposed mixture model to specific geotechnical and geomechanical processes. The developed modeling framework is unique on national as well on international level and offers great potential for future research. The latter is motivated by the assumptions and restrictions associated with the present research. For example, the incorporation of mass and momentum transfer between the constituents would be of great practical relevance because geomechanical problems are often driven by local drainage and consolidation phenomena as well as by contact constraints. 
Acknowledgments. The authors gratefully acknowledge the financial support by the German Research Foundation (DFG; Grant SA 310/26-2) as part of the DFG Research Unit FOR 1136. The authors are also grateful to their colleagues in this research unit for collaboration and for helpful discussions.

\section{Nomenclature}

\section{Operators and Special Notations}

$\cdot,:, \otimes$ single contraction, double contraction, tensor product

$\langle\cdot\rangle \quad$ spatial average

$(\cdot),(\cdot)^{\alpha}$ material time derivative, of an $\alpha$-related field

(.) referential, ALE description

$(\cdot)^{\alpha k} \quad \alpha k$-intrinsic average

$(\cdot)$ objective rate

$\boldsymbol{\nabla}(\cdot) \quad$ covariant derivative, gradient

$\partial(\cdot) \quad$ boundary, partial derivative

$\operatorname{div}(\cdot)$ divergence

$\Delta(\cdot) \quad$ increment

$\operatorname{tr}(\cdot) \quad$ trace of a second-order tensor

\section{Superscripts and Subscripts}

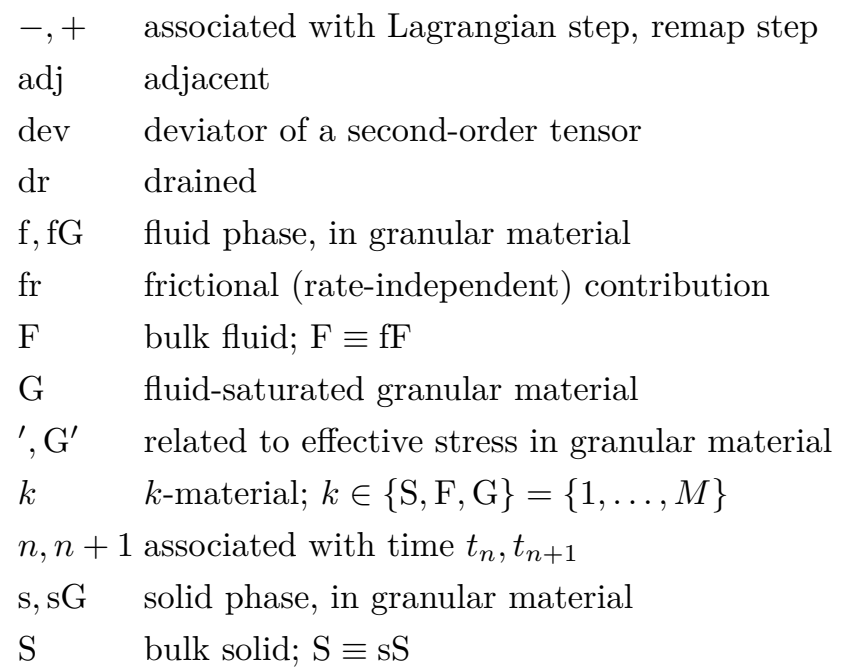




$\begin{array}{ll}\mathrm{T} & \text { transpose of a tensor } \\ \text { uj } & \text { unjacketed } \\ \text { vi } & \text { viscous (rate-dependent) contribution } \\ \alpha & \alpha \text {-phase; } \alpha \in\{\mathrm{s}, \mathrm{f}\}=\{1, \ldots, N\} \\ \alpha k & \alpha \text {-phase in } k \text {-material; } \alpha k \in\{\mathrm{S}, \mathrm{F}, \mathrm{sG}, \mathrm{fG}\}\end{array}$

\section{Latin Symbols}

$\begin{array}{ll}\boldsymbol{b}, \boldsymbol{b}^{\alpha k},\langle\boldsymbol{b}\rangle & \text { body force per unit mass } \\ \boldsymbol{B} & \text { strain operator matrix } \\ \boldsymbol{c} & \text { convective velocity } \\ \boldsymbol{c} & \text { fourth-order material tangent tensor } \\ \boldsymbol{C} & \text { damping matrix } \\ d & \text { line constant } \\ \boldsymbol{d}, \boldsymbol{d}^{k} & \text { spatial rate of deformation } \\ \mathrm{d} a, \mathrm{~d} v & \text { surface area density, volume density } \\ \boldsymbol{D} & \text { material stiffness matrix } \\ \mathcal{D} & \text { modeling domain in the ambient space } \\ f, f^{k}, f^{\alpha k} & \text { volume fractions, of } k, \alpha k \\ \boldsymbol{f}^{\text {in }}, \boldsymbol{f}^{\text {ex }} & \text { vector of internal, external nodal forces } \\ \boldsymbol{f}^{b}, \boldsymbol{f}^{\boldsymbol{t}} & \text { vector of body, surface forces } \\ H, H^{k}, H^{\alpha k} & \text { volume measures of } \mathcal{H}, \mathcal{H}^{k}, \mathcal{H}^{\alpha k} \\ \mathcal{H} & \text { representative volume element }(\mathrm{RVE}) \\ \mathcal{H}^{k}, \mathcal{H}^{\alpha k} & \text { portions of } k, \alpha k \text { in } \mathcal{H} \\ I & \text { local node, vertex, edge } \\ \boldsymbol{I} & \text { second-order unit tensor } \\ J & \text { Jacobian } \\ K, K^{k}, K^{\alpha k},\langle K\rangle & \text { bulk modulus } \\ \boldsymbol{K} & \text { effective stiffness matrix } \\ l_{\text {micro }}, l_{\text {meso }}, l_{\text {macro }} & \text { microscale, mesoscale, macroscale } \\ M & \text { mass, number of materials in the mixture } \\ \boldsymbol{M} & \text { mass matrix } \\ n, n^{k}, n^{\mathrm{G}} & \text { fluid fraction, porosity } \\ \boldsymbol{n} & \text { normal on interface } \\ N & \text { number of phases in the mixture } \\ & \end{array}$




$\begin{array}{ll}\boldsymbol{N}_{p}, \boldsymbol{N}_{\boldsymbol{v}} & \text { matrix of the interpolation functions } \\ p^{\alpha k}, p^{k},\langle p\rangle & \text { pressure } \\ p^{\prime}, p^{\mathrm{G}^{\prime}} & \text { mean effective stress } \\ \boldsymbol{p} & \text { nodal pressure vector } \\ q, q^{k}, q^{\alpha k} & \text { generic spatial field } \\ \boldsymbol{Q} & \text { coupling matrix } \\ \boldsymbol{r} & \text { vector of residuals } \\ \boldsymbol{s}^{k}, \boldsymbol{s}^{\alpha k},\langle\boldsymbol{s}\rangle & \text { extra stress } \\ \boldsymbol{S} & \text { compressibility matrix } \\ t & \text { time } \\ \boldsymbol{u} & \text { nodal displacement vector } \\ \boldsymbol{v}, \boldsymbol{v}^{k}, \boldsymbol{v}^{\alpha k},\langle\boldsymbol{v}\rangle & \text { spatial velocity } \\ V & \text { volume, of an element } \\ \boldsymbol{w} & \text { mesh velocity } \\ \boldsymbol{x} & \text { point in the ambient space } \\ \boldsymbol{y} & \text { vector of nodal degrees of freedom }\end{array}$

\section{Greek Symbols}

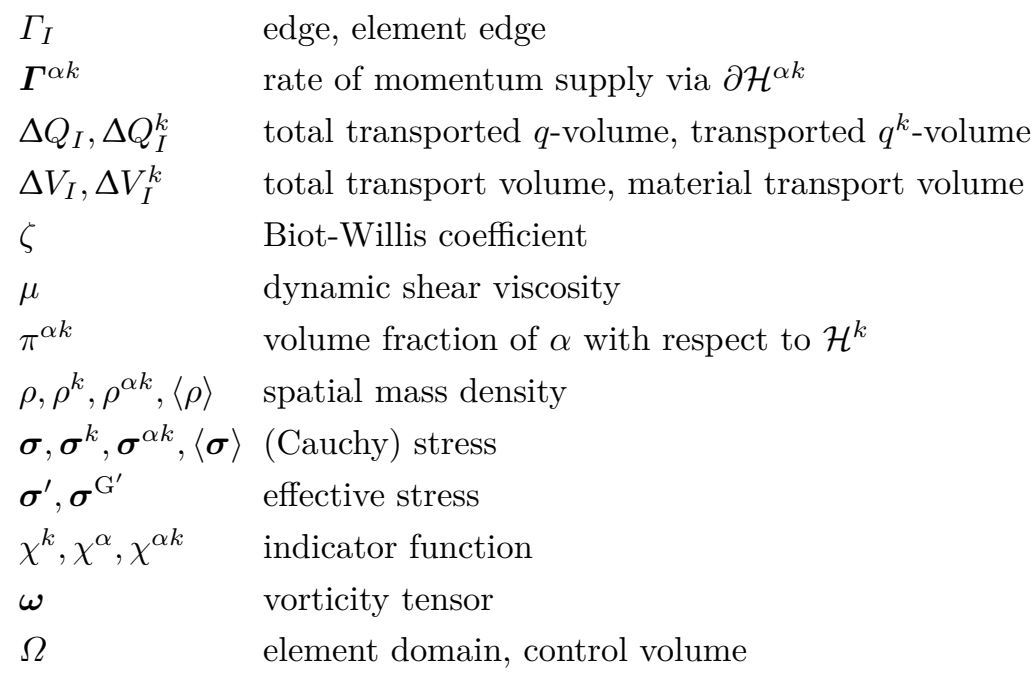




\section{Acronyms}

$\begin{array}{ll}\text { ALE } & \text { Arbitrary Lagrangian-Eulerian } \\ \text { CFD } & \text { Computational Fluid Dynamics } \\ \text { FEM } & \text { Finite Element Method } \\ \text { MMALE } & \text { Multi-Material Arbitrary Lagrangian-Eulerian } \\ \text { MPM } & \text { Material Point Method } \\ \text { MOF } & \text { Moment Of Fluid } \\ \text { PIV } & \text { Particle Image Velocimetry } \\ \text { RVE } & \text { Representative Volume Element } \\ \text { SPH } & \text { Smoothed Particle Hydrodynamics } \\ \text { VOF } & \text { Volume Of Fluid }\end{array}$

\section{References}

1. S. Abadie, D. Morichon, S. Grilli, and S. Glockner. Numerical Simulation of Waves Generated by Landslides using a Multiple-Fluid Navier-Stokes Model. Coastal Engineering, 57:779-794, 2010.

2. S. Achanta, J. H. Cushman, and M. R. Okos. On Multicomponent, Multiphase Thermomechanics with Interfaces. International Journal of Engineering Science, 32(11):1717-1738, 1994.

3. F. L. Addessio, J. R. Baumgardner, J. K. Dukowicz, N. L. Johnson, B. A. Kashiwa, R. M. Rauenzahn, and C. Zemach. CAVEAT: A Computer Code for Fluid Dynamics Problems with Large Distortion and Internal Slip. Report LA-10613-MS-REV. 1 (revised edition), Los Alamos National Laboratory, Los Alamos, USA, 1990.

4. R. J. Adrian. Particle-Imaging Techniques for Experimental Fluid Mechanics. Annual Review of Fluid Mechanics, 23:261-304, 1991.

5. C. Ancey, P. Coussot, and P. Evesque. A Theoretical Framework for Granular Suspensions in a Steady Simple Shear Flow. Journal of Rheology, 43(6):1673-1699, 1999.

6. C. Ancey and P. Evesque. Frictional-Collisional Regime for Granular Suspension Flows Down an Inclined Channel. Physical Review E, 62(6):8349-8360, 2000.

7. C. Ancey. Dry Granular Flows Down an Inclined Channel: Experimental Investigations on the Frictional-Collisional Regime. Physical Review E, 65(011304), 2001.

8. C. Ancey. Plasticity and Geophysical Flows: A Review. Journal of Non-Newtonian Fluid Mechanics, 142:4-35, 2007.

9. K. G. Anderson and R. Jackson. A Comparison of the Solutions of Some Proposed Equations of Motion of Granular Materials for Fully Developed Flow Down Inclined Planes. Industrial \& Engineering Chemistry Fundamentals, 241:145-168, 1992.

10. T. Arbogast, J. Douglas, and U. Hornung. Derivation of the Double Porosity Model of Single Phase Flow via Homogenization Theory. SIAM Journal on Mathematical Analysis, 21(4):823-836, 1990. 
11. D. N. Arnold, F. Brezzi, and M. Fortin. A Stable Finite Element for the Stokes Equations. Calcolo, 21(4):337-344, 1984.

12. S. Assier Rzadkiewicz, C. Mariotti, and P. Heinrich. Numerical Simulation of Submarine Landslides and Their Hydraulic Effects. Journal of Waterway, Port, Coastal and Ocean Engineering, 123(4):149-157, 1997.

13. D. Aubram. Differential Geometry Applied to Continuum Mechanics. Number 44 in Veröffentlichungen des Grundbauinstitutes der Technischen Universität Berlin. Shaker Verlag, Aachen, 2009. http://dx.doi.org/10.14279/depositonce-2185

14. D. Aubram. An Arbitrary Lagrangian-Eulerian Method for Penetration into Sand at Finite Deformation. Number 62 in Veröffentlichungen des Grundbauinstitutes der Technischen Universität Berlin. Shaker Verlag, Aachen, 2013. http://dx.doi.org/ 10.14279/depositonce-3958

15. D. Aubram. Optimization-Based Smoothing Algorithm for Triangle Meshes over Arbitrarily Shaped Domains. arXiv e-prints, 1410.5977 [cs.NA], 2014. http://arxiv . org/abs/1410.5977

16. D. Aubram. Development and Experimental Validation of an Arbitrary Lagrangian-Eulerian (ALE) Method for Soil Mechanics. Geotechnik, 38(3):193-204, 2015. http://dx.doi.org/10.1002/gete. 201400030

17. D. Aubram. Homogeneous Equilibrium Model for Geomechanical Multi-Material Flow with Compressible Constituents. Journal of Non-Newtonian Fluid Mechanics, 232:88-101, 2016. http://dx.doi.org/10.1016/j.jnnfm.2016.04.001

18. D. Aubram. A Multi-Material Eulerian Method for Large Deformation and Free Surface Flow of Geomaterials. (in preparation).

19. D. Aubram, F. Rackwitz, and S. A. Savidis. An ALE Finite Element Method for Cohesionless Soil at Large Strains: Computational Aspects and Applications. In T. Benz and S. Nordal (eds.): Proceedings 7th European Conference on Numerical Methods in Geotechnical Engineering (NUMGE), pages 245-250. CRC Press, Boca Raton, 2010.

20. D. Aubram, F. Rackwitz, P. Wriggers, and S. A. Savidis. An ALE Method for Penetration into Sand Utilizing Optimization-Based Mesh Motion. Computers and Geotechnics, 65:241-249, 2015. http://dx.doi.org/10.1016/j.compgeo.2014.12. 012

21. D. Aubram, F. Rackwitz, and S. A. Savidis. Vibro-Injection Pile Installation in Sand: Part I-Interpretation as Multi-Material Flow. In Th. Triantyfyllidis (ed): Holistic Simulation of Geotechnical Installation Processes: Numerical and Physical Modelling, pages 73-102. Springer International Publishing, 2015. http: //dx.doi.org/10.1007/978-3-319-18170-7_5

22. D. Aubram, S. A. Savidis, and F. Rackwitz. Theory and Numerical Modeling of Geomechanical Multi-Material Flow. In Th. Triantyfyllidis (ed): Holistic Simulation of Geotechnical Installation Processes: Benchmarks and Simulations, pages 187-229. Springer International Publishing, 2016. http://dx.doi.org/10. 1007/978-3-319-23159-4_10

23. R. A. Bagnold. Experiments on a Gravity-Free Dispersion of Large Solid Spheres in a Newtonian Fluid Under Shear. Proceedings of the Royal Society of London. Series A, 225:49-63, 1954.

24. W. Bai. The Quadrilateral 'Mini' Element for the Stokes Problem. Computer Methods in Applied Mechanics and Engineering, 143:41-47, 1997.

25. S. G. Bardenhagen, J. U. Brackbill, and D. Sulsky. The Material-Point Method for Granular Materials. Computer Methods in Applied Mechanics and Engineering, $187: 529-541,2000$ 
26. A. Bedford and D. S. Drumheller. Theories of Immiscible and Structured Mixtures. International Journal of Engineering Science, 21(8):863-960, 1983.

27. T. Belytschko, W. K. Liu, and D. Moran. Nonlinear Finite Elements for Continua and Structures. John Wiley \& Sons, Ltd., 2000.

28. L. S. Bennethum. Compressibility Moduli for Porous Materials Incorporating Volume Fraction. Journal of Engineering Mechanics, 132:1205-1214, 2006.

29. L. S. Bennethum. Theory of Flow and Deformation of Swelling Porous Materials at the Macroscale. Computers and Geotechnics, 34:267-278, 2007.

30. L. S. Bennethum and J. H. Cushman. Multiscale, Hybrid Mixture Theory for Swelling Systems-I. Balance Laws. International Journal of Engineering Science, 34(2):125-145, 1996

31. L. S. Bennethum and J. H. Cushman. Multiscale, Hybrid Mixture Theory for Swelling Systems-II. Constitutive Theory. International Journal of Engineering Science, 34(2):147-169, 1996.

32. L. S. Bennethum and T. Weinstein. Three Pressures in Porous Media. Transport in Porous Media, 54:1-34, 2004.

33. D. J. Benson. An Efficient, Accurate, Simple ALE Method for Nonlinear Finite Element Programs. Computer Methods in Applied Mechanics and Engineering, 72:305350, 1989.

34. D. J. Benson. Computational Methods in Lagrangian and Eulerian Hydrocodes. Computer Methods in Applied Mechanics and Engineering, 99:235-394, 1992.

35. D. J. Benson. A Multi-Material Eulerian Formulation for the Efficient Solution of Impact and Penetration Problems. Computational Mechanics, 15:558-571, 1995.

36. D. J. Benson. A Mixture Theory for Contact in Multi-Material Eulerian Formulations. Computer Methods in Applied Mechanics and Engineering, 140:59-86, 1997.

37. D. J. Benson. Volume of Fluid Interface Reconstruction Methods for MultiMaterial Problems. Applied Mechanics Reviews, 55(2):151-165, 2002.

38. D. J. Benson. An Implicit Multi-Material Eulerian Formulation. International Journal for Numerical Methods in Engineering, 48:475-499, 2000.

39. D. J. Benson. Momentum Advection On Unstructured Staggered Quadrilateral Meshes. International Journal for Numerical Methods in Engineering, 75:1549-1580, 2008.

40. D. J. Benson and S. Okazawa. Contact in a Multi-Material Eulerian Finite Element Formulation. Computer Methods in Applied Mechanics and Engineering, 193:42774298, 2004

41. L. Beuth, Z. Wieckowski, and P. A. Vermeer. Solution of Quasi-Static Large-Strain Problems by the Material Point Method. International Journal for Numerical and Analytical Methods in Geomechanics, 35:1451-1465, 2011.

42. M. A. Biot. General Theory of Three-Dimensional Consolidation. Journal of Applied Physics, 12:155-164, 1941.

43. M. A. Biot and D. G. Willis. The Elastic Coefficients of the Theory of Consolidation. Journal of Applied Mechanics, 24:594-601, 1957.

44. J. A. Bouré. Two-Phase Flow Models: The Closure Issue. Multiphase Science and Technology, 3(1-4):3-30, 1987.

45. J. A. Bouré and J. M. Delhaye. General Equations and Two-Phase Flow Modeling Section 1.2 in G. Hetsroni (ed.): Handbook of Multiphase Systems. Hemisphere Publishing Corporation, 1982.

46. R. M. Bowen. Theory of Mixtures. In A. C. Eringen (ed.): Continuum Physics. Volume III: Mixtures and EM Field Theories, part I. Academic Press, New York, 1976. 
47. R. M. Bowen. Incompressible Porous Media Models by Use of the Theory of Mixtures. International Journal of Engineering Science, 18(9):1129-1148, 1980.

48. R. M. Bowen. Compressible Porous Media Models by Use of the Theory of Mixtures. International Journal of Engineering Science, 20(6):697-735, 1982.

49. H. H. Bui, R. Fukagawa, K. Sako, and S. Ohno. Lagrangian Meshfree Particles Method (SPH) for Large Deformation and Failure Flows of Geomaterial using ElasticPlastic Soil Constitutive Model. International Journal for Numerical and Analytical Methods in Geomechanics, 32:1537-1570, 2008.

50. J. P. Carter, J. R. Booker, and E. H. Davis. Finite Deformation of an Elasto-plastic Soil. International Journal for Numerical and Analytical Methods in Geomechanics, 1:25-43, 1977.

51. C. H. Chang and J. D. Ramshaw. Dynamical Evolution of Volume Fractions in Multipressure Multiphase Flow Models. Physical Review E, 77(066305), 2008.

52. A. J. Chorin, T. J. R. Hughes, M. F. McCracken, and J. E. Marsden. Product Formulas and Numerical Algorithms. Communications on Pure and Applied Mathematics, 31:205-256, 1978.

53. P. Colella, H. M. Glaz, and R. E. Ferguson. Multifluid Algorithms for Eulerian Finite Difference Methods. 1997 (unpublished manuscript).

54. P. Coussot and C. Ancey. Rheophysical Classification of Concentrated Suspensions and Granular Pastes. Physical Review E, 59(4):4445-4457, 1999.

55. R. F. Craig. Craig's Soil Mechanics. E \& FN Spon, London, New York, 7th ed., 2007.

56. J. H. Cushman, L. S. Bennethum, and B. X. Hu. A Primer on Upscaling Tools for Porous Media. Advances in Water Resources, 25:1043-1067, 2002.

57. B. M. Das. Advanced Soil Mechanics. Taylor \& Francis, USA, 3rd ed., 2008.

58. Dassault Systèmes. Abaqus Analysis User's Guide, Version 6.14. Dassault Systèmes, 2014.

59. R. B. DeBar. Fundamentals of the KRAKEN Code. Technical Report UCID-17366, Lawrence Livermore Laboratory, Livermore, USA, 1974.

60. R. P. Denlinger and R. M. Iverson. Flow of Variably Fluidized Granular Masses across Three-Dimensional Terrain. 2. Numerical Predictions and Experimental Tests. Journal of Geophysical Research, 106(B1):553-566, 2001.

61. Y. Di, J. Yang, and T. Sato. An Operator-Split ALE Model for Large Deformation Analysis of Geomaterials. International Journal for Numerical and Analytical Methods in Geomechanics, 31:1375-1399, 2007.

62. DIN EN ISO 14688-1. Geotechnische Erkundung und Untersuchung - Benennung, Beschreibung und Klassifizierung von Boden - Teil 1: Benennung und Beschreibung. Beuth Verlag, Berlin, January 2003 (German Code).

63. D. A. Drew. Mathematical Modeling of Two-Phase Flow. Annual Review of Fluid Mechanics, 15:261-291, 1983.

64. D. A. Drew and S. L. Passman. Theory of Multicomponent Fluids. Springer, New York, 1999.

65. V. Dyadechko and M. Shashkov. Moment-of-Fluid Interface Reconstruction. Report LA-UR-05-7571, Los Alamos National Laboratory, Los Alamos, USA, 2005.

66. V. Dyadechko and M. Shashkov. Reconstruction of Multi-Material Interfaces from Moment Data. Journal of Computational Physics, 227:5361-5384, 2008.

67. F. Emeriault, B. Cambou, and A. Mahboubi. Homogenization for Granular Materials: Non Reversible Behaviour. Mechanics of Cohesive-Frictional Materials, 1:199 218, 1996.

68. Y. Forterre and O. Pouliquen. Flows of Dense Granular Media. Annual Review of Fluid Mechanics, 40:1-24, 2008. 
69. D. Freßmann. On Single- and Multi-Material Arbitrary Lagrangian-Eulerian Approaches with Application to Micromechanical Problems at Finte Deformations. Dissertation, Fachbereich Bauingenieur- und Vermessungswesen, Universität Hannover, Germany, 2004.

70. D. Freßmann and P. Wriggers. Advection Approaches for Single-and Multi-Material Arbitrary Lagrangian-Eulerian Finite Element Procedures. Computational Mechanics, 39:153-190, 2007.

71. S. Galera, J. Breil, and P.-H. Maire. A 2D unstructured multi-material CellCentered Arbitrary Lagrangian-Eulerian (CCALE) scheme using MOF interface reconstruction. Computers \& Fluids, 46:237-244, 2011.

72. R. A. Gingold and J. J. Monaghan. Smoothed Particle Hydrodynamics: Theory and Application to Non-Spherical Stars. Monthly Notices of the Royal Astronomical Society, 181:375-389, 1977.

73. G. Gudehus. A Comprehensive Constitutive Equation for Granular Materials. Soils and Foundations, 36(1):1-12, 1996.

74. M. Hassanizadeh and W. G. Gray. General Conservation Equations for MultiPhase Systems: 1. Averaging Procedure. Advances in Water Resources, 2:131-144, 1979.

75. M. Hassanizadeh and W. G. Gray. General Conservation Equations for MultiPhase Systems: 2. Mass, Momenta, Energy, and Entropy Equations. Advances in Water Resources, 2:191-203, 1979.

76. M. Hassanizadeh and W. G. Gray. General Conservation Equations for MultiPhase Systems: 3. Constitutive Theory for Porous Media Flow. Advances in Water Resources, 3:25-40, 1980.

77. M. Hassanizadeh and W. G. Gray. Mechanics and Thermodynamics of Multiphase Flow in Porous Media including Interphase Boundaries. Advances in Water Resources, 13(4):169-186, 1990.

78. P. Heinrich. Nonlinear Water Waves Generated by Submarine and Aerial Landslides. Journal of Waterway, Port, Coastal and Ocean Engineering, 118(3):249-266, 1992.

79. M. A. Hicks, J. Dijkstra, M. Lloret-Cabot, and M. Karstunen (eds.). Installation Effects in Geotechnical Engineering. CRC Press, London, 2013.

80. Ch. Hirsch. Numerical Computation of Internal and External Flows, Volume 1: Fundamentals of Computational Fluid Dynamics. Butterworth-Heinemann, Burlington, MA, USA, 2nd ed., 2007.

81. C. W. Hirt and B. D. Nichols. Volume of Fluid (VOF) Method for the Dynamics of Free Boundaries. Journal of Computational Physics, 39:201-225, 1981.

82. C. W. Hirt, A. A. Amsden, and J. L. Cook. An Arbitrary Lagrangian-Eulerian Computing Method for all Flow Speeds. Journal of Computational Physics, 14:227253, 1974.

83. U. Hornung. Homogenization and Porous Media. Springer-Verlag Berlin, 1997.

84. Y. Hu and M. F. Randolph. A Practical Numerical Approach for Large Deformation Problems in Soil. International Journal for Numerical and Analytical Methods in Geomechanics, 22:327-350, 1998.

85. T. J. R. Hughes. Numerical Implementation of Constitutive Models: RateIndependent Deviatoric Plasticity. In S. Nemat-Nasser, R. J. Asaro, and G. A. Hegemier (eds.): Theoretical Foundation for Large-Scale Computations for Nonlinear Material Behavior, pages 29-63. Martinus Nijhoff Publishers, Dordrecht, Niederlande, 1984.

86. K. Hutter and K. R. Rajagopal. On Flows of Granular Materials. Continuum Mechanics and Thermodynamics, 6:81-139, 1994. 
87. K. Hutter, B. Svendsen, and D. Rickenmann. Debris Flow Modeling: A Review. Continuum Mechanics and Thermodynamics, 8:1-35, 1996.

88. J. M. Hyman. Numerical Methods for Tracking Interfaces. Physica D 12:396-407, 1984.

89. M. Ishii and T. Hibiki. Thermo-Fluid Dynamics of Two-Phase Flow. Springer Science+Business Media, LLC, 2nd ed., 2011.

90. R. M. Iverson. The Physics of Debris Flows. Reviews of Geophysics, 35(3):245-296, 1997.

91. R. M. Iverson. The Debris-Flow Rheology Myth. In D. Rickenmann and C. L. Chen (eds): Debris-Flow Hazards Mitigation: Mechanics, Prediction, and Assessment, pages 303-314. Millpress, Rotterdam, 2003.

92. R. M. Iverson and R. P. Denlinger. Flow of Variably Fluidized Granular Masses Across Three-Dimensional Terrain. 1. Coulomb Mixture Theory. Journal of Geophysical Research, 106(B1):537-552, 2001.

93. P. C. Johnson and R. Jackson. Frictional-Collisional Constitutive Relations for Granular Materials, with Application to Plane Shearing. Journal of Fluid Mechanics, 176:67-93, 1987.

94. P. Jop, Y. Forterre, and O. Pouliquen. A Constitutive Law for Dense Granular Flows. Nature, 441:727-730, 2006.

95. D. Kolymbas. Introduction to Hypoplasticity. A. A. Balkema, Rotterdam, 2000.

96. P. V. Lade and R. de Boer. The Concept of Effective Stress for Soil, Concrete and Rock. Géotechnique, 47:61-78, 1997.

97. P. V. Lade and J. A. Yamamuro (eds). Physics and Mechanics of Soil Liquefaction. A. A. Balkema, Rotterdam, The Netherlands, 1999.

98. R. J. LeVeque. Finite Volume Methods for Hyperbolic Problems. Cambridge University Press, Cambridge, UK, 3rd ed., 2002.

99. R. W. Lewis and B. A. Schrefler. The Finite Element Method in the Static and Dynamic Deformation and Consolidation of Porous Media. John Wiley \& Sons, Chichester, 2nd ed., 1998.

100. X. S. Li. A Sand Model with State-Dependent Dilatancy. Géotechnique, 52(3):173-186, 2002.

101. Livermore Software Technology Corporation (LSTC). LS-DYNA Keyword User's Manual, Volume I, R8.0. Livermore Software Technology Corporation (LSTC), 2015.

102. D. S. Liyanapathirana. Arbitrary Lagrangian Eulerian based Finite Element Analysis of Cone Penetration in Soft Clay. Computers and Geotechnics, 36:851-860, 2009.

103. J. Locat and H. J. Lee. Submarine Landslides: Advances and Challenges. Canadian Geotechnical Journal, 39:193-212, 2002.

104. I. Loges and A. Niemunis. Neohypoplasticity - Estimation of Small Strain Stiffness. In Th. Triantyfyllidis (ed): Holistic Simulation of Geotechnical Installation Processes: Numerical and Physical Modelling, pages 163-180. Springer International Publishing, 2015.

105. L. B. Lucy. A Numerical Approach to the Testing of the Fission Hypothesis. The Astronomical Journal, 82:1013-1024, 1977.

106. G. Luttwak and R. L. Rabie. The Multi Material Arbitrary Lagrangian Eulerian Code MMALE and Its Application to Some Problems of Penetration and Impact. Technical Report LA-UR-85-2311, Los Alamos National Laboratory, Los Alamos, New Mexico, USA, 1985.

107. G. Ma, F. Shi, and J. T. Kirby. Shock-Capturing Non-Hydrostatic Model for Fully Dispersive Surface Wave Processes. Ocean Modelling, 43-44:22-35, 2012.

108. G. Ma, J. T. Kirby, and F. Shi. Numerical Simulation Of Tsunami Waves Generated By Deformable Submarine Landslides. Ocean Modelling, 69:146-165, 2013. 
109. M. E. Mabsout and J. L. Tassoulas. A Finite Element Model for the Simulation of Pile Driving. International Journal for Numerical Methods in Engineering, 37:257278, 1994.

110. H. U. Mair. Review: Hydrocodes for Structural Response to Underwater Explosions. Shock and Vibration, 6:81-96, 1999.

111. L. E. Malvern. Introduction to the Mechanics of a Continuous Medium. Prentice Hall, Inc., New Jersey, 1969.

112. D. A. Mandell, T. F. Adams, K. S. Holian, F. L. Addessio, J. R. Baumgardner, and S. J. Mosso. MESA: A 3-D Computer Code for Armor/Anti-Armor Applications. Report LA-UR-89-1851, Los Alamos National Laboratory, Los Alamos, USA, 1989.

113. M. T. Manzari and Y. F. Dafalias. A Critical State Two-Surface Plasticity Model for Sands. Géotechnique, 47(2):255-272, 1997.

114. D. G. Masson, C. B. Harbitz, R. B. Wynn, G. Pedersen, and F. Løvholt. Submarine Landslides: Processes, Triggers and Hazard Prediction. Philosophical Transactions of the Royal Society of London. Series A, 364:2009-2039, 2006.

115. J. M. McGlaun and S. L. Thompson. CTH: A Three-Dimensional Shock Wave Physics Code. International Journal of Impact Engineering, 10:351-360, 1990.

116. G. H. Miller and E. G. Puckett. A High-Order Godunov Method for Multiple Condensed Phases. Journal of Computational Physics, 128:134-164, 1996.

117. D. S. Miller and G. B. Zimmerman. An Algorithm for Time Evolving Volume Fractions in Mixed Zones in Lagrangian Hydrodynamics Calculations. Russian Journal of Physical Chemistry B, 3:117-121, 2009.

118. M. P. Moseley and K. Kirsch (eds). Ground Improvement. Spon Press, New York, 2nd ed., 2004.

119. M. A. Murad, L. S. Bennethum, and J. H. Cushman. A Multi-Scale Theory of Swelling Porous Media: I. Application to One-Dimensional Consolidation. Transport in Porous Media, 19:93-122, 1995.

120. M. A. Murad and J. H. Cushman. Multiscale Flow and Deformation in Hydrophilic Swelling Porous Media. International Journal of Engineering Science, 34(3):313-338, 1996.

121. M. Nazem, D. Sheng, and J. P. Carter. Stress Integration and Mesh Refinement for Large Deformation in Geomechanics. International Journal for Numerical Methods in Engineering, 65:1002-1027, 2006.

122. M. Nazem, D. Sheng, J. P. Carter, and S. W. Sloan. Arbitrary LagrangianEulerian Method for Large-Strain Consolidation Problems. International Journal for Numerical and Analytical Methods in Geomechanics, 32(9):1023-1050, 2008.

123. A. Niemunis and I. Herle. Hypoplastic Model for Cohesionless Soils with Elastic Strain Range. Mechanics of Cohesive-Frictional Materials, 2:279-299, 1997.

124. A. Niemunis, C. E. Grandas Tavera, and T. Wichtmann. Peak Stress Obliquity in Drained and Undrained Sands. Simulations with Neohypoplasticity. In Th. Triantyfyllidis (ed): Holistic Simulation of Geotechnical Installation Processes: Benchmarks and Simulations, pages 85-114. Springer International Publishing, 2016.

125. M. A. Nikolinakou, A. J. Whittle, S. A. Savidis, and U. Schran. Prediction and Interpretation of the Performance of a Deep Excavation in Berlin Sand. Journal of Geotechnical and Geoenvironmental Engineering, 137(11):1047-1061, 2011.

126. W. F. Noh. CEL: A Time-Dependent, Two-Space-Dimensional, Coupled EulerianLagrange Code. In B. Alder et al. (eds): Methods in Computational Physics. Advances in Research and Applications. Volume 3: Fundamental Methods in Hydrodynamics, pages 117-179. Academic Press, New York and London, 1964.

127. S. L. Passman, J. W. Nunziato, P. B. Bailey, and K. W. Reed. Shearing Motion of a Fluid-Saturated Granular Material. Journal of Rheology, 30(1):167-192, 1986. 
128. M. Pastor, O. C. Zienkiewicz, and A. H. C. Chan. Generalized Plasticity and the Modelling of Soil Behaviour. International Journal for Numerical and Analytical Methods in Geomechanics, 14:151-190, 1990.

129. J. S. Peery and D. E. Carroll. Multi-Material ALE Methods in Unstructured Grids. Computer Methods in Applied Mechanics and Engineering, 187:591-619, 2000.

130. E. B. Pitman and L. Le. A Two-Fluid Model for Avalanche and Debris Flows. Philosophical Transactions of the Royal Society of London. Series A, 363:1573-1601, 2005.

131. O. A. Plumb and S. Whitaker. Dispersion in Heterogeneous Porous Media. 1: Local Volume Averaging and Large-Scale Averaging. Water Resources Research, 24(7):913-926, 1988

132. S. P. Pudasaini. A General Two-Phase Debris Flow Model. Journal of Geophysical Research, 117(F03010), 2012.

133. S. P. Pudasaini and K. Hutter. Avalanche Dynamics: Dynamics of Rapid Flows of Dense Granular Avalanche. Springer-Verlag Berlin Heidelberg, 2007.

134. S. P. Pudasaini, Y. Wang, and K. Hutter. Modelling Debris Flows Down General Channels. Natural Hazards and Earth Systems Sciences, 5:799-819, 2005.

135. G. Qiu, S. Henke, and J. Grabe. Application of a Coupled Eulerian-Lagrangian Approach on Geomechanical Problems Involving Large Deformations. Computers and Geotechnics, 38:30-39, 2011.

136. F. Rackwitz and S. A. Savidis. Numerische Untersuchungen zum Tragverhalten von Zugpfählen in Berliner Sand. Bauingenieur, 79(9):375-383, 2004. (in German)

137. W. J. Rider and D. B. Kothe. Reconstructing Volume Tracking. Journal of Computational Physics, 141:112-152, 1998.

138. M. Rudman. Volume-Tracking Methods for Interfacial Flow Calculations. International Journal for Numerical Methods in Fluids, 24(7):671-691, 1997.

139. S. B. Savage and K. Hutter. The Motion of a Finite Mass of Granular Material Down a Rough Incline. Journal of Fluid Mechanics, 199:21-24, 1989.

140. S. A. Savidis, D. Aubram, and F. Rackwitz. Arbitrary Lagrangian-Eulerian Finite Element Formulation for Geotechnical Construction Processes. Journal of Theoretical and Applied Mechanics, 38(1-2):165-194, 2008.

141. S. A. Savidis, D. Aubram, and F. Rackwitz. Vibro-Injection Pile Installation in Sand: Part II-Numerical and Experimental Investigation. In Th. Triantyfyllidis (ed): Holistic Simulation of Geotechnical Installation Processes: Numerical and Physical Modelling, pages 103-131. Springer International Publishing, 2015. http://dx.doi. org/10.1007/978-3-319-18170-7_6

142. R. Scardovelli and S. Zaleski. Direct Numerical Simulation of Free-Surface and Interfacial Flow. Annual Review of Fluid Mechanics, 31:567-603, 1999.

143. M. Shashkov. Closure Models for Multimaterial Cells in Arbitrary LagrangianEulerian Hydrocodes. International Journal for Numerical Methods in Fluids 56(8):1497-1504, 2008.

144. A. N. Schofield and C. P. Wroth. Critical State Soil Mechanics. McGraw-Hill, New York, 1968.

145. R. B. Seed, K. O. Cetin, R. E. S. Moss, A. M. Kammerer, J. Wu, J. M. Pestana, M. F. Riemer, R. B. Sancio, J. D. Bray, R. E. Kayen, and A. Faris. Recent Advances in Soil Liquefaction Engineering: A Unified and Consistent Framework. Technical Report EERC 2003-06, University of California, Berkeley, California, USA, 2003.

146. D. Sheng, M. Nazem, and J. P. Carter. Some Computational Aspects for Solving Deep Penetration Problems in Geomechanics. Computational Mechanics, 44:549-561, 2009. 
147. J. C. Simo and T. J. R. Hughes. Computational Inelasticity. Springer-Verlag Berlin, 1998.

148. K. Soga, E. Alonso, A. Yerro, K. Kumar, and S. Bandara. Trends in LargeDeformation Analysis of Landslide Mass Movements with Particular Emphasis on the Material Point Method. Géotechnique, 66(3):248-273, 2016.

149. H. B. Stewart and B. Wendroff. Two-Phase Flow: Models and Methods. Journal of Computational Physics, 56:363-409, 1984.

150. D. Sulsky, S.-J. Zhou, and H. L. Schreyer. Application of a Particle-In-Cell Method to Solid Mechanics. Computer Physics Communications, 87:236-252, 1995.

151. E. Susila and R. D. Hryciw. Large Displacement FEM Modelling of the Cone Penetration Test (CPT) in Normally Consolidated Sand. International Journal for Numerical and Analytical Methods in Geomechanics, 27:585-602, 2003.

152. M. Taiebat and Y. F. Dafalias. SANISAND: Simple Anisotropic Sand Plasticity Model. International Journal for Numerical and Analytical Methods in Geomechanics, 32:915-948, 2008.

153. K. Terzaghi. Theoretical Soil Mechanics. John Wiley \& Sons, Inc., New York, 1943.

154. K. K. Tho and C. F. Leung and Y. K. Chow and S. Swaddiwudhipong. Eulerian Finite-Element Technique for Analysis of Jack-Up Spudcan Penetration. International Journal of Geomechanics, 12:64-73, 2012.

155. Th. Triantafyllidis (ed). Numerical Modelling of Construction Processes in Geotechnical Engineering for Urban Environment. CRC Press, London, 2006.

156. Th. Triantafyllidis (ed). Holistic Simulation of Geotechnical Installation Processes: Numerical and Physical Modelling. Vol. 77 in Lecture Notes in Applied and Computational Mechanics. Springer International Publishing, 2015. http: //dx.doi.org/10.1007/978-3-319-18170-7

157. Th. Triantafyllidis (ed). Holistic Simulation of Geotechnical Installation Processes: Benchmarks and Simulations. Vol. 80 in Lecture Notes in Applied and Computational Mechanics. Springer International Publishing, 2016. http://dx.doi.org/ 10.1007/978-3-319-23159-4

158. C. Truesdell and R. A. Toupin. Encyclopedia of Physics. Bd. III/1: The Classical Field Theories, pages 226-793. Springer-Verlag Berlin Göttingen Heidelberg, 1960.

159. C. Truesdell and W. Noll. The Non-Linear Field Theories of Mechanics. SpringerVerlag Berlin Heidelberg New York, 3rd ed., 2004.

160. J. G. Trulio and K. R. Trigger. Numerical Solution of the One-Dimensional Hydrodynamic Equations in an Arbitrary Time-Dependent Coordinate System. Report UCRL-6522, Lawrence Radiation Laboratory, University of California, Livermore, USA, 1961.

161. J. G. Trulio. Theory and Structure of the AFTON Codes. Report AFWL-TR66-19, Air Force Weapons Laboratory, Kirtland Air Force Base, New Mexico, USA, 1966.

162. J. Vogelsang, G. Huber, and T. Triantafyllidis. Requirements, Concepts, and Selected Results for Model Tests on Pile Penetration. In Th. Triantyfyllidis (ed): Holistic Simulation of Geotechnical Installation Processes: Benchmarks and Simulations, pages 1-30. Springer International Publishing, 2016.

163. J. Vogelsang, G. Huber, T. Triantafyllidis, and T. Bender. Interpretation of Vibratory Pile Penetration Based on Digital Image Correlation. In Th. Triantyfyllidis (ed): Holistic Simulation of Geotechnical Installation Processes: Benchmarks and Simulations, pages 31-51. Springer International Publishing, 2016. 
164. P.-A. von Wolffersdorff. A Hypoplastic Relation for Granular Materials with a Predefined Limit State Surface. Mechanics of Cohesive-Frictional Materials, 1:251271, 1996.

165. W. N. Weseloh, S. P. Clancy, and J. W. Painter. PAGOSA Physics Manual. Report LA-14425-M, Los Alamos National Laboratory, Los Alamos, USA, 2010.

166. S. Whitaker. Flow in Porous Media I: A Theoretical Derivation of Darcy's Law. Transport in Porous Media, 1:3-25, 1986.

167. S. Whitaker. Flow in Porous Media II: The Governing Equations for Immiscible, Two-Phase Flow. Transport in Porous Media, 1:105-125, 1986.

168. S. Whitaker. Flow in Porous Media III: Deforming Media. Transport in Porous Media, 1:127-154, 1986.

169. S. Whitaker. The Method of Volume Averaging. Kluwer Academic Publishers, Dordrecht, 1999.

170. D. J. White and W. A. Take. GeoPIV: Particle Image Velocimetry (PIV) Software for Use in Geotechnical Testing. Technical Report CUED/D-SOILS/TR322, Geotechnical and Environmental Research Group, University of Cambridge, UK, 2002.

171. D. J. White, W. A. Take, and M. D. Bolton. Soil Deformation Measurement using Particle Image Velocimetry (PIV) and Photogrammetry. Géotechnique, 53(7):619631, 2003.

172. A. B. Wood. A Textbook of Sound. The Macmillan Company, New York, 1930.

173. D. M. Wood. Soil Mechanics: A One-Dimensional Introduction. Cambridge University Press, USA, 2009.

174. P. Wriggers. Nonlinear Finite Element Methods. Springer-Verlag Berlin Heidelberg, 2008.

175. D. L. Youngs. Time-Dependent Multi-Material Flow with Large Fluid Distortion, pages 273-285. In K. W. Morton und M. J. Baines (eds.): Numerical Methods for Fluid Dynamics. Academic Press, London, 1982.

176. O. C. Zienkiewicz, A. H. C. Chan, M. Pastor, B. A. Schrefler, and T. Shiomi. Computational Geomechanics - With Special Reference to Earthquake Engineering. John Wiley \& Sons, Chichester, 1999.

177. O. C. Zienkiewicz and R. L. Taylor. The Finite Element Method. Volume 1: The Basis. Butterworth-Heinemann, 5th ed., 2000. 